\title{
„in der aller bessten formb“: Adlige Testamente der 1560er Jahre
}

\section{Einleitung}

Die folgende Untersuchung basiert auf insgesamt 14 Testamenten ${ }^{1}$ aus dem Bestand des Niederösterreichischen Landmarschallsamtes, die in Form und Umfang erheblich variieren. Konkret handelt es sich um die Testamente der 60er Jahre des 16. Jahrhunderts, ${ }^{2}$ die in vielen Elementen den Einfluss des römischen Rechtes bzw. der gelehrten Juristen offenbaren. ${ }^{3}$

Der Bestand der Testamente des Niederösterreichischen Landmarschallamtes hat als solcher eine bewegte Geschichte: Grundsätzlich ist anzumerken, dass die Zuständigkeit für die $\mathrm{Ab}$ handlung der Testamente nach dem Erblasser

\footnotetext{
${ }^{1}$ Vgl. zum Begriff OGRIS, Testament 152f. („[...] ist eine einseitige letztwillige Verfügung, der (bezeugte) Letzte Wille des Erblassers").

${ }^{2}$ Dazu ist anzumerken, dass das Testament der Eufemia von Stubenberg aus dem Jahr 1557 zusätzlich aufgenommen wurde, da einerseits kein verfälschend großer Zeitabstand zum im Fokus stehenden Jahrzehnt besteht und andererseits innerhalb der getroffenen Auswahl das Testament ihres Ehemannes Casimir zu Polheim von 1563 vorhanden ist und damit vielfältige Vergleichsmöglichkeiten eröffnet werden. Ebenfalls aus dem Rahmen fällt das Testament der Barbara Pösing, da das Hauptstück zwar vom 10. 8. 1568 stammt, aber 1571 und 1574 insgesamt zwei Ergänzungen durch die Testatorin selbst vorgenommen wurden (vgl. die Tabelle).

${ }^{3}$ Zur Rezeption KIEFNER, Rezeption (privatrechtlich) 970-984; zum Einfluss des kanonischen Testaments bzw. Klerikertestaments OGRIS, Testament 154f.
}

bzw. der Erblasserin bestimmt wurde, ${ }^{4}$ für die Landstände, also u.a. die landsässigen adligen Personen (Herren, Ritter), sowie in Niederösterreich auch für die Beamten der Stände das Landrecht (Niederösterreichisches Landmarschallamt) verantwortlich war. In Österreich ob der Enns war der Landeshauptmann, Vertreter des Landesfürsten, Vorsitzender des Landrechtes, im Land unter der Enns das Haupt der Stände, der Landmarschall. Beisitzer waren in der untersuchten Zeit Landräte des Herren- und Ritterstandes. ${ }^{5}$ Hinzuweisen ist auf die Niederösterreichische Regierung, die die höchste Verwaltungs- und Gerichtsinstanz in Österreich ob und unter der Enns war. Wichtig war deren Rolle als zweite Instanz nach dem ständischen Landrecht. ${ }^{6}$ Schwierigkeiten gab es, wenn mehrere Gerichtsinstanzen zuständig sein konnten, was auch in den untersuchten Testamenten beobachtet werden kann, wobei der jeweilige Testator bzw. in den untersuchten Fällen ausschließlich eine Testatorin explizit den Gerichtsstand in einer strittigen Situation festlegte (s. unten). Für die Aufbewahrung der Unterlagen hatten die Reformen in der zweiten Hälfte des 18. Jahrhunderts eine Konzentration beim k. k. Nieder-

\footnotetext{
${ }^{4}$ HochedLINGER, Archivarischer Vandalismus 291293.

${ }^{5} \mathrm{Zu}$ Kompetenzen der Landrechte und deren Geschichte Ebd. 305-314.

${ }^{6}$ Nächste Instanz war der Geheime Rat, von dort bis 1637 der Reichshofrat. Zu den Kompetenzen und der Geschichte der Niederösterreichischen Regierung EBD. 300-304.
} 
österreichischen Landrecht zur Folge. Auch der Bestand des Landmarschallischen Gerichts gelangte in den 1760er Jahren dorthin. ${ }^{7}$ Nach den Reformen der Mitte des 19. Jahrhunderts wurden die Unterlagen im Landesgerichtsarchiv im Wiener Justizpalast aufbewahrt, das sich „zum Mekka der Familien- und Personenforschung"8 entwickelte. Von dort gelangte der Bestand des Niederösterreichischen Landmarschallamtes nicht unwidersprochen im Zuge der Auflösung dieser Institution 1924 mit der Begründung der vielfachen Übereinstimmung der Familienzugehörigkeit der Erblasser mit dem Obersthofmarschallamt in das Haus-, Hof- und Staatsarchiv. ${ }^{9}$

Aufgrund der hohen Zahl an Testamenten können diese leider nicht in allen, äußerst interessanten Aspekten vorgestellt werden, so wäre ein genauer Vergleich der eingesetzten formelhaften Wendungen und damit verbundene Abhängigkeiten von Interesse. Im Zentrum der Analyse stehen die Testamente, dann die Frage nach der in diesen angewandten Selbstbezeichnung, die Form der Stücke, die Darstellung des strukturellen Aufbaus der letzten Willen und schließlich die Hinweise zur Umsetzung der Testamente. Dadurch soll ein Schlaglicht auf diese wichtige Quellenform in der Mitte des 16. Jahrhunderts und den Umgang mit den letzten Dingen in der adligen Gesellschaft geworfen werden. Da sich diese Untersuchung auf adlige Testamente beschränkt, also auf Stücke von Testatorinnen und Testatoren aus dem Herren- und Ritterstand oder zumindest mit einer Nähe zu diesen, etwa wenn eine Familie kurz darauf in diesen Stand aufgenommen wurde, werden die Verweise auf Jany Partisan zwar vergleichend angeführt, doch gilt $\mathrm{zu}$ betonen, dass dieser keinem der beiden zugewiesen werden konnte.

\footnotetext{
7 Ebd. 309f., 322f.

${ }^{8}$ Ebd. 325.

${ }^{9}$ Ebd. 325-343. bes. 334f., 337, 339-342.
}

\section{Die Testamente ${ }^{10}$}

Betrachtet man zunächst Vorgaben zur Errichtung eines Testaments in der Landtafel von Österreich ob der Enns, ${ }^{11}$ so kannte diese für die rechtmäßige Erklärung des freien Willen zur Handhabung des Erbes nach dem Tod zwei Möglichkeiten, nämlich die schriftliche (solenne in scriptis) und mündliche (nuncupativum) Form. Testierfähig war prinzipiell jeder, sofern dazu

${ }^{10}$ An den Beginn sei eine Anmerkung zur Zitierweise der behandelten Schriftstücke gestellt: Die entsprechenden Stücke werden in Tabelle 1 aufgelistet, wo auch die jeweilige Archivsignatur samt Abkürzungsschlüssel angegeben ist. Da diese durchgehend keine Foliierung bzw. Paginierung aufweisen, ein allgemeines Zitat bei einem mehrere Seiten umfassenden Stück aber relativ unbrauchbar ist, wird im folgenden eine „virtuelle“ Foliierung vorgenommen, d.h. die erste Seite des Schreibtextes des Testaments wird als $1^{\mathrm{r}}$ zitiert und so weiter. Leerseiten am Beginn des Textes werden also nicht einbezogen, auf die beiliegenden Schrifttexte oder Umschläge kann aufgrund des überschaubaren Inhalts allgemein verwiesen werden. Bei den Urkunden erfolgt der genauere Verweis durch die Angabe der entsprechenden Zahl der Verfügung, was aber nur beim letzten Willen von Eufemia zu Polheim möglich ist. Bei Wolf Streun zu Schwarzenau muss diese Angabe unterbleiben.

${ }^{11}$ In der Folge bezieht sich diese Arbeit immer wieder auf diese gesetzlichen Vorgaben aus der ersten Hälfte des 17. Jahrhunderts, auch wenn diese etwas jünger sind und nicht dem Land unter der Enns galten, doch können sie als Rahmen, Richtlinie oder Möglichkeiten verstanden werden, anhand derer Unterschiede und Übereinstimmungen bei den untersuchten Testamenten umso klarer hervortreten (zu Österreich ob der Enns vgl. kompakt WESENER, Erbrecht 15f.). Für das Land unter der Enns war neben einem unter Ferdinand I. entstandenen Entwurf einer Landesordnung ein solcher 1573 von dem niederösterreichischen Regimentsrat Dr. Püdler von großer Bedeutung, in dem er sich auf geltendes Recht stützte. Dieser wurde vom Landesfürsten zwar nicht offiziell anerkannt, fand aber in der Praxis große Beachtung. 1595 vollendeten Reichart Freiherr von Schwarzenau und Dr. Linsmayer eine Überarbeitung der Landesordnung. Ein 1654 entstandener Entwurf einer Landesordnung lässt einen stärkeren Einfluss des römischen Rechtes erkennen (WESENER, Erbrecht 13-15). 
tauglich, also bei geistiger Gesundheit (Sanamente-Formel). Das Testament musste zudem mit freiem Willen verfasst werden, worauf neben der Betonung der geistigen Gesundheit in den vorliegenden Stücken auch regelmäßig hingewiesen wurde. Ausgeschlossen waren unvogtbare Personen, die Altersgrenze lag hier bei 18 bzw. 20, bei Frauen bei 16 Jahren. Mit einer Heirat erreichte man automatisch die Vogtbarkeit. Eingeschränkt war die Testierfähigkeit u.a. bei Personen, die stumm und taub waren. Letzte Vertreter des männlichen Stammes konnten allein jene Güter vererben, die sie selbst erworben hatten oder ihr natürlicher Erbteil waren. Daher entfielen Güter, die durch Verzicht von weiblichen Verwandten an diese gelangten. ${ }^{12}$ Daran wird bereits deutlich, dass der Nachlass eines Erblassers oder einer Erblasserin unterschiedliche Qualitäten haben bzw. in Sondervermögen (Fahrnis, Kaufgut, Erbgut, Heergerät etc.) zerfallen konnte und demnach gewisse Einschränkungen in der Testierfreiheit vorhanden waren. ${ }^{13}$

Insgesamt beschäftigt sich die Studie mit 13 adligen Testamenten, wobei sieben Testatorinnen und Testatoren dem Herren- und sechs dem Ritterstand zugewiesen werden können. Fünf Testamente wurden von Männern, acht von Frauen verfasst, wobei angemerkt werden muss, dass das Schriftstück der Eufemia von Stubenberg außerhalb des eigentlich interessierenden Jahrzehnts liegt. Zugleich unterscheiden sich die Testamente in ihrer äußeren Form: Die 14 Dokumente unterteilen sich in zwei Pergamenturkunden, zwei gebundene Libelle und schließlich zehn aus Papierbögen in Folioformat zusam-

${ }^{12}$ Vgl. zum ultimus familiae auch WESENER, Erbrecht 125f. Verzicht der Tochter: Ebd. 84-98; STRÄTZ, Landtafel Titel 2f., 317-320. Vgl. zur Testierfähigkeit kurz OGRIS, Testament 160f.

${ }^{13}$ Vgl. etwa OGRIS, Nachlaß 820-823; Ogris, Noterben 1056-1059; WESENER, Erbrecht 21-23. Zum römischen Recht Hausmaniger, SelB, Römisches Privatrecht, 348-350. mengelegte Stücke, bei denen sich teilweise auch die Fadenbindung erhalten hat. Neben Testamenten begegnen auch Kodizille, was bereits auf den Einfluss des gemeinen Rechtes verweist. Der wesentliche Unterschied beider Formen ist, dass erstere nach römischem Recht eine ausdrückliche Erbeinsetzung enthalten mussten. ${ }^{14}$

Eine genaue Darstellung der Familienverhältnisse, v.a. auch im Bezug auf die im Testament bedachten Verwandten, kann im folgenden nicht geboten werden, da diese für die Fragestellung dieser Untersuchung weniger relevant sind. Einige zusätzliche Informationen bietet die Tabelle, wodurch die Verhältnisse der Testatorinnen und Testatoren deutlicher gemacht werden sollen. Noch einige Anmerkungen zur Namenspraxis bei den Frauentestamenten: Eufemia bezeichnete sich zu Beginn als geborene Stubenberg und Frau des Casimir zu Polheim und Wartenburg, in ihrer eigenhändigen Unterfertigung lautet der Zusatz nach dem Geburtsnamen allein Frau zu Polheim. Die Nennung des Mädchennamens an erster Stelle und dann die des Ehemannes zu Beginn des letzten Willens erfolgt auch bei Anna Kannibal, Margaretha Prockh und bei Barbara Pösing. Catharina Steghofer, eine geborene Stubmer, nennt nicht nur den Geburtsnamen, sondern den beider Eltern, um dann den des aktuellen Ehemannes folgen $\mathrm{zu}$ lassen. ${ }^{15}$ Alle unterfertigten aber, sofern erhalten, zumindest mit dem Nachnamen des Ehegatten, Catharina Steghofer setzte laut der erhaltenen Abschrift geborene Stubmer hinzu. Fehlt die Unterschrift, wie bei Anna Kannibal, dann bezeichnete sie sich als solche in der Corroboratio.

\footnotetext{
${ }^{14} \mathrm{Vgl}$. zum Kodizill den rechtlichen Rahmen bei STRÄTZ, Landtafel Titel 25, 361-363. Zum römischen Recht Hausmaniger, SelB, Römisches Privatrecht 348. ${ }^{15}$ Dies mag auch damit zusammenhängen, dass die Stubmer eine Ritterfamilie sind, während ihr Mann, Stefan Steghofer, erst nach ihrem Tod in den Adelsstand aufgenommen wurde (s. Tabelle).
} 
Bei Apollonia Perner und Regina Zelking erfolgte die Namensnennung in umgekehrter Reihenfolge, also etwa: Apollonia des Herrn Niklas Perner Frau, eine geborene Puchheim. Regina Zelking wiederholt nach dem eigenen Namen mit dem Zusatz geborene Lamberg dann den des Ehemannes. Elisabeth Nagl nannte allein den Nachnamen ihres Ehemannes Caspar. Dieser Namenspraxis der Unterschriften, sofern erhalten, der Corroboratio oder der Intitulatio soll auch im folgenden Rechnung getragen werden.

\section{Selbstbezeichnung, Testamentsformen}

Als Selbstbezeichnung der Dokumente ist beinahe durchgängig jene als Testament zu finden. ${ }^{16}$ Oft erscheint der Begriff in Verbindung mit anderen Klassifikationen, wie Geschäft, Ordnung, Meinung, Befehl, testamentliche Ordnung/Institution oder v.a. auch letzter (endlicher) Wille. Das Auftreten ist unterschiedlich, es erfolgte zumeist eine Nennung zu Beginn, oft, aber nicht ausschließlich im Rahmen der Arenga und bei den rechtserheblichen Formeln im $\mathrm{Zu}$ sammenhang mit der Rechtswirksamkeit, die auch am Anfang, vermehrt aber am Ende der Testamente auftreten. Für die einleitenden Formulierungen sei beispielhaft die Formulierung bei Mang Irnfried zitiert, der das Schriftstück als "ordnung unnd testament" oder auch als "tesstament unnd lesten willen ${ }^{\prime 17}$ bezeichnete. Bei dessen Bestellung der Testamentsexekutoren erfolgte die immer wieder charakteristische dreimalige Wiederholung der synonym gebrauchten Begriffe, wobei Zusammenstellung und Verwendung in den einzelnen letzten Willen variieren: „mein lester will, testament unnd ordnung". ${ }^{18}$ Diese Wiederholungen hatten si-

\footnotetext{
${ }^{16}$ Ausnahme: Anna Kannibal.

17 Irnfried, $1^{\mathrm{r}}$.

${ }^{18}$ Irnfried, $4^{\mathrm{r}}$.
}

cherlich die Funktion einer Erhöhung der Rechtssicherheit. ${ }^{19}$ Seltener wurden lateinische Bezeichnungen zur Klassifizierung der Schriftstücke verwendet, Casimir zu Polheim bezeichnete seine Urkunde etwa als "dise mein ordnung, tesstament, geschäfft unnd lessten willen $\mathrm{zu}$ latein testamentum solemne oder in scriptis". ${ }^{20}$ Ebenso taucht eine Nennung in Zusammenhang mit der Sicherstellung der Rechtswirksamkeit auf: Diese Formeln können einerseits zu Beginn, aber auch am Ende auftreten, wie noch beim Kapitel zur Struktur der Schriftstücke genauer darzustellen sein wird. Konkret wurde darin einerseits die Rechtsnorm des Testaments definiert, andererseits bei Nichtwirksamkeit des Schriftstückes aufgrund eines formalen Fehlers oder anderen Mangels eine Einschränkung vorgenommen, damit die Verfügungen zumindest auf einer niedrigeren Rechtsstufe Geltung haben könnten (favor testamenti, z. B. als Kodizill, Schenkung von Todes wegen, ${ }^{21}$ etc.). Beispielhaft seien die entsprechenden Passagen im Testament von Casimir zu Polheim zitiert, der am Ende des Protokolls nach der Sana-mente-Formel festhielt, dass er das Schriftstück „in der aller bessten formb, weiß unnd mainung, aines lessten willens, wie das nach ordnung der rechten, der tesstament, codicil unnd aines yeden anndern lessten willens, allem gebrauch nach vor hohen unnd nidern gerichten, sonnderlich dises lanndts Ossterreich ob der Enns am aller crefftigissten unnd bestendigissten immer sein soll, beschehen khan unnd mag, thue unnd gethan haben will“. ${ }^{22}$ Typischer, weil beinahe immer vorhanden, sind die Formeln zu Sicherstellung

\footnotetext{
${ }^{19}$ Vgl. etwa Polheim, $1^{\mathrm{r}}$ (Testament und letzter Wille) $1^{\mathrm{v}}$.

${ }^{20}$ Polheim, $1^{\mathrm{v}}$. Ein testamentum solenne sollte vor sieben Zeugen und einem kaiserlichen Notar errichtet werden (WESENER, Erbrecht 133. 137).

${ }^{21}$ Siehe dazu STRÄTZ, Landtafel Titel 32, 265f. und zum römischen Recht HAUSMANINGER, SELB, Römisches Privatrecht 364.

${ }^{22}$ Polheim, $1^{\mathrm{v}}$.
} 
der Rechtswirksamkeit am Ende der Testamente, wie es auch bei Casimir zu Polheim geschieht: "Unnd ist dem allem nach mein beschliesslicher, entlicher lesster will, mainung unnd bevelch, sover unnd wo diß mein tesstament aus abganng oder mengl, zugehoriger notturfftiger zierlichait oder annderer gebrechen halber, wie die erdacht oder genanndt werden mechten, in selbigem wesen alls ain schrifftlich, zierlich tesstament $\mathrm{zu}$ uncrefften verfellt unnd gebracht wurde, das es doch alsdann volkhumene wurkhliche crafft haben unnd behallten solle alls ein nuncupatioum, mundlich oder vom mund ausgesprochen, tesstament oder alls ein codicill, alls ain gab von todts wegen beschehen, genannt causa mortis, oder sonnst alls ain yeder annder bestendiger lesster will, alls fideicomiss ${ }^{23}$ unnd dergleichen allergestallt unnd in albege, alls weren all notturfftig form, gezirden, wesentliche stukh, clausen unnd requisit unnd expresse wie sich geburt vergriffen und gesezt.“24 Auch seine Ehefrau Eufemia von Polheim verwendete solche juristischen Fachausdrücke. ${ }^{25}$ Zusätzlich erfolgte bei Casimir zu Polheim die Nennung von möglichen Selbstbezeichnungen des Schriftstückes auch bei der Formel des Widerrufsrechtes (worunter in dieser Arbeit die Klausel für die Möglichkeit zur Errichtung eines neuen Testaments oder von Zusätzen verstanden wird ${ }^{26}$ ), in der er sich ex-

${ }^{23}$ Zum Fideikommiss im römischen Recht: HAusMANIGER, SELB, Römisches Privatrecht 362-364.

${ }^{24}$ Polheim, $6^{\mathrm{r}}$.

${ }^{25}$ Stubenberg: „[...] alls solcher lesster willen, genannt testamentum codicil nuncupatioum [...]“; "solches mein testament und lezter will, auch crafft unnd macht habe alls ain codicil iure codicilorum". Steghofer, $4^{\mathrm{r}}$ : "codicill, geschäfft, ubergab oder donation vonn todts weegen".

${ }^{26}$ OGRIS, Testament 152f., 161f.; zum Widerruf auch WESENER, Erbrecht 153-155. Festzuhalten ist, dass die ältere Vergabung von Todes wegen eine rechtliche Bindung bewirkte, ein Widerruf von Testamenten erst durch den Einfluss des römischen Rechtes üblich, ein plizit die Möglichkeit eines „codicils, donation, legats, fideicommiss oder dergleichen lessten willens“27 einräumte. In der Beglaubigung bezeichnete er seinen letzten Willen dann als Libell, bezog sich somit auf die äußere Form. ${ }^{28}$ Ein interessantes Beispiel stellen auch die Verfügungen Barbara Pösings in Form eines drei Schriftstücke umfassenden Libells dar. Sie hatte sich in ihrem Testament vorbehalten, dieses selbst aufzuheben, abzuändern oder durch Beilagen zu erweitern. ${ }^{29}$ Ein Verweis zu Beginn des letzten Willens belegt, dass sie von diesem Recht bereits Gebrauch gemacht hatte, indem sie die Außerkraftsetzung eines Testaments aus dem Jahr 1559 betonte. ${ }^{30}$ Immer wieder verwies sie auch auf mögliche Änderungen durch ein künftiges Kodizill. ${ }^{31}$ Am Ende ihres Geschäfts erneuerte sie den Vorbehalt mit folgenden Worten: ,[...] doch will ich mir in disen allein vorbehalten, do ich an bahren gelt oder andern meinen aigenthumblichen haab und güttern etwaß uber diß mein testament verordnen, verschaffen oder verschreiben, auch dasselb nur mit meinen tauffnamb und aigener handt underschriben und mit sigel oder pettschadt von mir verferttigt oder an statt meiner handtschrifft neben mein

Widerrufsverzicht sogar als ungültig betrachtet wurde, WESENER, Erbrecht 153.

${ }^{27}$ Polheim, $6^{\text {r }}$.

${ }^{28}$ Polheim, $8^{\mathrm{r}}$.

${ }^{29}$ Pösing, $1^{\mathrm{v}}$.

${ }^{30}$ Pösing, 1v: „Und ob ainerley ander geschäfft, ordnung oder testament hievor, es sey lang oder khurz, von mir ausgangen und am dato elter oder disen meinen gegenwertigen lezten willen und geschäfft widerwerttig nach meinem abgang ruchbahr oder fürtragen wurden, so will ich solche und sonderlich, daß ich zu Wienn im neun und fünffzigistn jahr aufgericht, hiemit wissentlich widerruft haben, daz diselben abgethan, cassirt und vernicht werden sollen, alß ob die nie außgangen noch aufgericht wern."

${ }^{31}$ Pösing, 6r , 9v. Bei der ersten Stelle wurde explizit auf ein "hernach aufgericht codicill” verwiesen, vielleicht wurden also bei den Formeln 1571 auch im Haupttestament Veränderungen vorgenommen, da diese Verweise 1568 noch wenig Sinn hatten. 
pettschadt, zween wissentlich bestendig erber zeugen fürgestelt wurden, daß solches in allen besten formb und maß, alß were solches alles und jedes insonderheit mit allen und jeden zierlichen nothwendigen puncten, artikheln, namen und gebührlichen clausln in diß mein testament von wortt zu wortt austrükhlichen eigeleibt und eingeschriben.“ ${ }^{32}$ Wichtig ist diese Passage auch, da die Testatorin die für künftige Abänderungen zur Rechtssicherung notwendigen Kriterien aufzählte, also eigenhändige Unterschrift und Petschaft oder bei Fehlen dieser die Unterfertigung zweier Zeugen neben der eigenen Petschaft. Dabei hat sie sich wohl an dem gültigen Rechtsbrauch orientiert. ${ }^{33}$ Tatsächlich unterfertigte Barbara Pösing die Erweiterung ihres letzten Willens eigenhändig und fügte ihr Petschaft bei, benannte zusätzlich noch zwei Zeugen, die ebenfalls unterschrieben und ihr Siegel anbrachten. ${ }^{34}$ Für die Frage der Selbstbezeichnung ist nun interessant, wie Barbara Pösing die beiden folgenden Schriftstücke bezeichnete. In dem von 1571 stammenden Schriftstück wird zu Beginn auf das Testament von 1568 verwiesen, in dem sie sich vorbehalten hatte, dieses "durch ain codicill oder andere schrifften under meiner handtschrifft, sigill oder pettschadt zu mindern, zu mehren, zuverkheren oder gahr aufzuheben, wie ich verlust", diese "minderung, mehrung, veränderung, erkhlärung meines offtgedachten testament ${ }^{\prime \prime 35}$ würde sie nun vornehmen. Im folgenden Schriftstück verwies sie wiederum auf den letzten Willen von 1568, gleichzeitig aber

32 Pösing, 14r . Eine solche Ergänzung durch Kodizill, aber nicht eine Erbeinsetzung oder Enterbung entsprach dem Landesbrauch, WESENER, Erbrecht 156.

${ }^{33}$ Dieser sah zudem die Wirksamkeit auch bei einem Zeugensiegel vor. Bei Fehlen jeder Fertigung des Erblassers waren zwei (Siegel) bzw. drei (Petschaft) Zeugen notwendig, bei einer Frau zwei bzw. vier, WESENER, Erbrecht 155. Diese Unterscheidung ist vor dem Hintergrund der Verfügung Barbara Pösings bemerkenswert.

${ }^{34}$ Pösing, $18^{v}$.

${ }^{35}$ Pösing, $15^{\mathrm{r}}$. auch auf das 1571 verfasste Schriftstück, das sie als Deklaration oder Kodizill bezeichnete. Diese Möglichkeit einer Beilage mit Änderungen zum Testament in Form eines Kodizills wurde in der Landtafel von Österreich ob der Enns explizit erwähnt. ${ }^{36} 1574$ war die Verordnung und das Geschäft, wie sie es selbst bezeichnete, notwendig geworden, da ein Erbe vor ihr verstorben war und sie den dadurch frei werdenden Geldbetrag nun auf andere Weise verteilte. ${ }^{37}$ Diese Erklärung bestätigte Barbara Pösing wiederum selbst mit ihrer Petschaft, benannte diesmal sogar drei Zeugen, die ebenfalls jeweils unterschrieben und ihre Siegel beifügten. ${ }^{38}$ Dieses Beispiel wurde deshalb so ausführlich dargestellt, da es die unterschiedlichen und mehrfachen Begriffe sowie deren Verwendung für die verschiedenen Dokumenttypen deutlich machen kann, zusätzlich wichtige Informationen für die Frage der Rechtsgültigkeit solcher Verfügungen bietet.

Zusammengefasst gilt es auf die oftmalige Wiederholung der Selbstbezeichnungen innerhalb der Texte, aber auch auf die doppelte und dreifache Aufzählung dieser Begriffe bei den einzelnen Textpassagen hinzuweisen. Der Begriff des Testamentes ist somit insgesamt betrachtet am Beginn der zweiten Hälfte des 16. Jahrhunderts fest im Vokabular verankert, einzelne Stücke beinhalten darüber hinausgehend lateinische Fachausdrücke, die auf ein größeres juristisches Fachwissen im Hintergrund hindeuten, wobei hier v.a. die Testamente von Casimir und Eufemia zu Polheim herausstechen. Insgesamt haben all diese Formeln den Zweck, dem letzten Willen eine höhere Rechtssicherheit zu verleihen.

\footnotetext{
${ }^{36}$ STRÄTZ, Landtafel Titel 25, § 4f., 361. Dieses soll bei eigenhändiger Unterfertigung gültig sein (STRÄTZ, Landtafel Titel 25, §7, 362). Vgl. allgemein zu Änderungen STRÄTZ, Landtafel Titel 29, § 4f., 373f.

${ }^{37}$ Pösing, 19r-v .

${ }^{38}$ Pösing, 21 ${ }^{\mathrm{r}-\mathrm{v}}$.
} 


\section{Allgemeine Klassifikation der untersuchten Testamente}

Bevor die Struktur der Testamente dargestellt werden kann, muss auf die unterschiedlichen Überlieferungsformen der auf uns gekommenen Stücke verwiesen werden. Erwähnt seien zu Beginn die Rahmenbedingungen von schriftlichen Testamenten, wie sie in der Landtafel festgelegt wurden: Zum ersten war ein letzter Wille gültig, wenn dieser gänzlich in eigener Handschrift und mit Tauf- und Zunamen unterschrieben sowie durch Siegel ${ }^{39}$ oder Petschaft innen oder außen beglaubigt war. Wurde nur mit eigener Hand unterschrieben sowie Petschaft und Siegel angebracht, sollte das Testament nur gültig sein, wenn zwei Zeugen mit Siegel oder vier mit Petschaft innen oder außen dieses bestätigten, sowie mit Bittzetteln dazu aufgefordert worden waren. Konnten sie diese nicht vorlegen, mussten sie diese Bitte durch Eid bestätigen. Dem Landesbrauch im Land unter der Enns nach war nur ein Zeuge mit Siegel oder zwei mit Petschaft notwendig.40 Unterschrieb der Testator nur, so benötigte er zwei (drei) ${ }^{41}$ Zeugen mit Siegeln oder drei (fünf) mit Petschaft. Auch hier waren im Land unter der Enns zwei Zeugen mit Siegel oder vier mit Petschaft ausreichend. ${ }^{42}$ Unterfertigte er nicht eigenhändig, fehlte auch Siegel oder Petschaft, waren drei (vier) bzw. fünf (sieben) Zeugen notwendig. Im Land unter der Enns waren wiederum nur zwei Zeugen mit Siegel oder vier mit Petschaft notwendig. ${ }^{43}$ Verfasste jemand das Testament eigenhändig und beglaubigte es mit

\footnotetext{
${ }^{39}$ In den Quellen stets als Insiegel bezeichnet.

${ }^{40}$ WesEneR, Erbrecht 132, verweist auf Püdler und Strein-Linsmayer.

${ }^{41}$ Die Angaben in Klammer beziehen sich auf Korrekturen durch die Fassung von 1629.

42 WESENER, Erbrecht 132, verweist auf Püdler und Strein-Linsmayer.

${ }^{43}$ WeSENER, Erbrecht 132, verweist auf Püdler und Strein-Linsmayer.
}

seiner Petschaft, so musste er die Zeugen nicht persönlich um Fertigung bitten oder dieses vortragen, sondern konnte es mit einem Bittzettel offen oder verschlossen zuschicken. Dies galt auch, wenn der Testator nur lesen konnte. Konnte jemand weder schreiben noch lesen, so musste er die Zeugen zu sich berufen oder zu ihnen kommen und ihnen seinen Willen mündlich vortragen. ${ }^{44}$ Besonders bemerkenswert ist die unterschiedliche Rechtsqualität von Siegel und Petschaft. Zusätzlich ist anzumerken, dass die Landtafel keine Unterschiede bei allographen Testamenten von Männern oder Frauen kannte, ganz im Gegensatz zum Land unter der Enns, wo diese jeweils ein Siegel oder zwei Petschafte mehr erbitten mussten. ${ }^{45}$ Zur Form sei abschließend angemerkt, dass an einer Stelle festgelegt wurde, dass Zahlenangaben ausgeschrieben werden sollten.6 Diese Praxis des

\footnotetext{
${ }^{44}$ Für schriftliche Testament STRÄTZ, Landtafel Titel 4, 320f.; Regelungen für mündliche letzte Willen vgl. zudem STRÄTZ, Landtafel Titel 5, 321f., sowie Fertigung mit Hilfe der Obrigkeit STRÄTZ, Landtafel Titel 6, 322-324.

${ }^{45}$ WESENER, Erbrecht 132, verweist auf Püdler und Strein-Linsmayer.

${ }^{46}$ STRÄTZ, Landtafel Titel 9, § 7, 327. Auch die Stadtordnung von Wien von 1526 unterschied für Männer bei einem eigenhändig verfassten Testament nicht zwischen Siegel und Petschaft, bei einem von anderer Hand verfassten Testament konnte der Erblasser mit seinem Siegel oder bei Fehlen von diesem durch seine Petschaft und ein Zeugensiegel oder zwei Zeugenpetschaften diesem Rechtsgültigkeit verleihen. Fehlte diesem auch eine Petschaft, dann musste einer an seiner Stelle sein Insiegel oder zwei ihre Petschaft anbringen. Konnte er das Testament weder schreiben noch unterschreiben, dann benötigte der Erblasser jemanden, der sein Siegel anbrachte, sowie zwei Zeugensiegel oder vier ehrbare Personen mit Petschaft. Bei Frauen, wobei nicht zwischen Verheirateten und Witwen unterschieden wird, waren drei Siegelführer oder fünf Männer mit Petschaft notwendig. Innerhalb dieses Abschnittes ist hervorzuheben, dass auch die Möglichkeiten von Jungfrauen und unter Vormundschaft stehenden unvogtbaren Kindern behandelt werden (CSENDES, Rechtsquellen 300-302). Für die Wiener Bürger gab es also unterschiedliche rechtliche
} 
Siegelns wird in den vorliegenden Stücken und damit ein Landesbrauch entgegen dem römischen Recht bestätigt, das eine Unterschrift vorsah. ${ }^{47}$

Aufgrund der äußeren Form der im Landmarschallamt vorliegenden Testamente wären allgemein zu unterscheiden: Abschriften (z.B. bei Apollonia Perner), beglaubigte Abschriften (Catharina Steghofer oder Barbara Pösing) und Ausfertigungen.

Bei den beglaubigten Abschriften ist der Vermerk des Schreibers am Ende des Textes von Interesse, der auch Aufschlüsse zum Umgang mit den Originalen gibt. So verfasste ein Sekretär des Landrechts (= Landgericht) unterhalb des Fließtextes des Testaments von Catharina Steghofer folgenden Kollationierungsvermerk: „Ist gegen den original collat[ioniert] und derselben gleich befunden. ${ }^{"}{ }^{48}$ Am Ende des letzten Willens wird dieser inhaltlich wiederholt, wobei der Schreiber darauf verwies, dass die Kontrolle bei der Landkanzlei erfolgt war. ${ }^{49}$ Im Fall Barbara Pösing ist diese Kollationierung ebenfalls gut nachvollziehbar: Am Ende des Testaments schrieb der Expeditor und Taxator der Landkanzlei: „Diese abschrifften allermassen solche hierinen zusamben gebunden, seind auf des lob[lichen] landtmarschallischen gerichts uber der jerozischer herrn curatorn deßwegen beschehenes anlangen undern dato zwainzigsten januarii des sechzehenhundert zwey und vierzig gethane gnedige verordnung von seinen drey absonderlichen wahren originalien bei der n[ieder]ö[sterreichsichen] landtcanzlei abcopirt, nachmahls durch mich underschribenen gegen demselben ordenlich gehalten, collationirt und

Vorgaben für Männer und Frauen. Deutlich wird damit auch, dass der Landesbrauch in Österreich unter der Enns dem der Wiener Stadtordnung entsprach. Vgl. allg. auch WESENER, Erbrecht 132-135.

${ }^{47}$ Dazu WeSENER, Erbrecht 137.

${ }^{48}$ Steghofer, $2^{\mathrm{r}}$.

${ }^{49}$ Steghofer, $4^{\mathrm{v}}$. corrigirtermassen gleichen inhalts bestunden, volgents wolgedachts lob[liches] g[eri]chts ambtsinsigel $\mathrm{zu}$ mehrer beglaubigung hiran gehangen worden. Actum landtcanzlei in Wienn den zehednen februarii anno ut supra"50. Abgesehen von den Aufschlüssen zum Umgang mit den Originalen, erfolgte in beiden Fällen die Abschrift und Beglaubigung innerhalb der Landkanzlei. Die Originale sind in beiden Fällen meines Wissens nach nicht erhalten. ${ }^{51}$

Die Originalausfertigungen lassen sich prinzipiell in eigenhändige und durch andere Hand verfasste Dokumente unterteilen, die folgende Kategorien bilden: ${ }^{2}$

1. Eigenhändige Ausfertigungen mit Unterschrift (ohne Zeugen!; z.B. Apollonia Perner, wobei es sich dabei um eine Abschrift handelt)

2. Eigenhändige Unterschrift mit Petschaft/Siegel (Zeugensiegel als Verschluss) ${ }^{53}$

3. Ausfertigung mit eigenhändiger Unterschrift, eigenem Petschaft/Siegel und nur Siegel von Zeugen

4. Ausfertigung mit eigenhändiger Unterschrift und Petschaft/Siegel sowie Siegel und Unterschrift der Zeugen

5. Ausfertigung nur mit Siegel: Nur Siegel der Zeugen (Anna Kannibal)

\footnotetext{
${ }^{50}$ Pösing, $21^{\mathrm{v}}$.

${ }^{51}$ Für das Testament von Barbara Pösing könnte der Verweis auf ein Dokument im "Preßburger Archiv“ ein Hinweis auf die Ausfertigung sein. Dieses Testament wurde laut den Angaben 1574 verfasst, was dem Datum des zweiten Kodizills entspricht (WERTNER, Grafen 234).

${ }^{52}$ Allgemein wäre zwischen mündlichen und schriftlichen Testamenten zu unterscheiden. Wesener unterteilt dann die schriftlichen Stücke in holographe (eigenhändige) und allographe Testamente. Insgesamt unterteilt er in drei Gruppen, nämlich in die eigenhändig verfassten, eigenhändig unterfertigten und jene Dokumente, bei denen jegliche Eigenhändigkeit fehlt (WESENER, Erbrecht 131f.).

${ }^{53}$ Der Landesbrauch akzeptierte diese Form als gleichwertige Unterfertigung (WESENER, Erbrecht 139).
} 
Vorausgeschickt sei kurz die Bezeichnung als Petschaft oder Siegel diskutiert, zumal das Landrecht diese explizit in ihrer Rechtsqualität unterschied (s. oben). Ein Petschaft konnte jedermann besitzen, ein Siegel nur ein „SiegelmäBiger “. ${ }^{54}$ In der Corroboratio wurde bei den Zeugen stets auf ihr Siegel, bei Anna Kannibal bei der Schadlosformel auf das Petschaft oder Siegel verwiesen. ${ }^{55}$ Das Anbringen des Petschaft der Zeugen wurde auch bei Elisabeth Nagl betont, die den Schlüsselamtmann in Krems sowie zwei Mitglieder des inneren Stadtrats von Krems dazu benannte. ${ }^{56}$ Die Testatoren selbst verwiesen auf ihr Petschaft ${ }^{57}$ oder aber auch auf das Siegel..$^{58}$ Betrachtet man diese Nennungen, fällt auf, dass alle Frauen mit dem Petschaft, die Männer mit ihrem Siegel den letzten Willen bestätigten. Ausnahmen bilden Barbara Pösing (Siegel) und Jany Partisan (Petschaft), ${ }^{59}$ was in beiden Fällen nicht verwundert, v.a. aber bei Pösing bemerkenswert ist. Diese Situation wurde bei Eufemia von Stubenberg ausformuliert, die festhielt, dass sie ein Petschaft habe verwenden müssen, da sie kein eigenes Siegel besitze. Die Zeugen beglaubigten auch mit ihren Siegeln, allein jene ausschließlich aus dem städtischen Bereich stammenden bei Elisabeth Nagl wurden um ihre Petschaft gebeten.

$\mathrm{Zu}$ der ersten Kategorie: Leider ist ein Testament mit diesen Rahmenbedingungen in dem Unter-

\footnotetext{
${ }^{54}$ WESENER, Erbrecht 131. Vgl. allgemein zum Unterschied WESENER, Erbrecht 131f.

${ }^{55}$ Der Begriff des Insiegels ist am Seitenrand nachträglich hinzugefügt (Kannibal, $2^{v}$ ).

${ }^{56}$ Nagl, $6^{\mathrm{r}-\mathrm{v}}$.

${ }^{57}$ Z.B. Stubenberg; Steghofer, $4^{\mathrm{r}}$; Prockh, 10r (Die Verweise in diesem Testament beziehen sich auf die beiliegende Abschrift, da diese durch die Abfassung auf einem kleineren Format und damit größere Übersichtlichkeit ein schnelleres Auffinden der entsprechenden Passagen ermöglicht.); Zelking, 4vi Nagl, 6ri Partisan, $3^{\mathrm{v}}$.

${ }^{58}$ Görtschach, 6r; Polheim, 8r; Irnfried, 5ri Pösing, 14" Pierbaum, $3^{\mathrm{v}}$; Schwarzenau.

${ }^{59}$ Pösing, 14v ; Partisan, $3^{\mathrm{v}}$.
}

suchungszeitraum nicht erhalten, doch erlaubt es die Corroboratio im Testament der Apollonia Perner ein solches zu erschließen. ${ }^{60}$ Diese lautet: „Und zu merer urkhundt habe ich dises testament mit aigner handt geschriben und auch mit aigner hand undergeschriben, auch mit meinem [8r] angepornnen pedtschafft verferttigt, darinnen ich mich für alle meine erben allen innhalt diß briefs bey meinen ehrn und trewen war und stett zuhalten verpinde." ${ }^{\prime 61}$ Es fehlt also ein Hinweis auf Zeugen für dieses Rechtsgeschäft, wodurch die Rechtswirksamkeit von eigenhändig ver- und unterfertigten Stücken bestätigt wird, sofern man nicht ein Auslassen der Formel vermuten will. ${ }^{62}$ Dies ist umso bemerkenswerter, da gerade eine Frau in diesem Fall auf die Beibringung von Zeugen verzichtete.

Die zweite Kategorie reduziert die Eigenhändigkeit auf die Unterschrift des Testators bzw. der Testatorin. Daneben erschien das Petschaft/Siegel, Zeugen traten allein zum Verschluss des Stückes auf. Als Beispiel kann hierfür der letzte Wille des Thomas Ott zu Pierbaum genannt werden, der als Ausfertigung erhalten ist. Diese wurde in einem mit vier Siegeln verschlossenen Kuvert aufbewahrt. Auf der letzten Seite des Testaments erscheint rechts neben dem Siegel folgende eigenhändige Unterfertigung: "Thoman Ott zu Pierpamb, mein aygne handt schrifft, damit ich obbegriffnen innhalt allen bezeig und bekhrefftig. " 63 Die gleichmäßige, zügige Linienführung verrät dabei eine geübte Hand. In der Corroboratio hatte er kurz zuvor die drei Zeugen genannt, darunter als letzten Caspar Nagl. Diese hatte er explizit durch einen

\footnotetext{
${ }^{60}$ Als früheres Beispiel vgl. etwa HHStA, NÖLMA 17, Nr. 40 (Wilhelm Kuenring) vom 29. 5. 1541.

${ }^{61}$ Perner, $7^{\mathrm{v}}-8^{\mathrm{r}}$.

${ }^{62}$ Hingewiesen sei darauf, dass bei diesem Stück ein Kollationierungsvermerk fehlt, allein das Rubrum auf der Rückseite bezeichnet dieses als Abschrift.

${ }^{63}$ Pierbaum, 4 .
} 
Bittzettel gebeten, das Testament neben seinem eigenen durch ihre Siegel zu verschließen.

Bei der dritten Kategorie umfasst die Beglaubigung die Unterschrift und das Petschaft/Siegel des Testators oder der Testatorin, sowie die Siegel der Zeugen. Eine solche Form hat das Testament des Hans von Görtschach zu Gross. Die vier Siegel sind deutlich zu erkennen, darunter die zittrige Unterschrift des Hans von Görtschach selbst, der darin auch seinen Stand als Ritter nannte. In der Corroboratio wurde ebenfalls darauf verwiesen, dass die drei Zeugen nur um die Anbringung des Siegels gebeten worden waren. ${ }^{64}$

Die vierte Kategorie umfasst sowohl Unterschrift und Petschaft/Siegel des Ausstellers, als auch jene der Zeugen. Diese Kategorie ist v.a. auch für den Bereich der Schriftlichkeit innerhalb der durch diese Testamente repräsentierten Adelsschicht von Interesse, indem dabei nicht nur die Testatoren selbst, sondern auch die Zeugen als Schriftkundige erscheinen. Eine solche Beglaubigungsform besitzt die erhaltene Ausfertigung des Testaments der Regina von Zelking. Diese und ihre drei Zeugen unterschrieben selbst. Die drei Siegel sollten laut Corroboratio neben der gewöhnlichen (!) Petschaft der Testatorin aufscheinen, befanden sich in diesem Fall aber nicht neben den Unterschriften, die direkt an die Datierung anschließen, sondern auf der Außenseite zum Verschließen des Dokumentes. ${ }^{65}$

Die fünfte Kategorie beschränkt sich auf das Testament der Anna Kannibal: In der Corroboratio wurde nur um die Siegel der Zeugen gebeten, eine eigene Unterfertigung der Testatorin selbst nicht erwähnt. Es erscheinen dann auch drei Siegel, wobei ein kleineres, schwarzes über diesen vielleicht jenes der Anna Kannibal selbst ist,

${ }^{64}$ Görtschach, 6rv-7r

65 Zelking, 4v. was aufgrund des Erhaltungszustands aber nicht $\mathrm{zu}$ beurteilen ist. ${ }^{66}$

Ein Sonderfall ist der letzte Wille Jany Partisans: In der Corroboratio erwähnte dieser nämlich explizit, dass er nicht schreiben könne, weshalb er einen im Land unter der Enns tätigen Sekretär aus dem Ritterstand gebeten habe, an seiner Stelle das Dokument zu unterfertigen. ${ }^{67}$ Er selbst hatte nur seine Petschaft aufgedrückt, zusätzlich drei Zeugen um deren Unterschrift und Siegel gebeten, die alle auf der Unterschriftenseite vorhanden sind. ${ }^{6}$

Hervorgehoben soll außerdem das mittlerweile durch mehrfache Verweise bereits wohlbekannte Testament des Casimir zu Polheim werden: Dieses liegt in Libellformvor, wird durch einen weißen Ledereinband geschützt und besitzt insgesamt noch drei anhangende Siegel. Besonders wird das Stück aber durch die Art der Beglaubigung: Nach der Datierung und damit nach dem Ende des eigentlichen letzten Willens folgt auf der gegenüberliegenden Seite die Unterfertigung des Testators selbst, die nicht allein aus der Unterschrift besteht, sondern in der er rechtserhebliche Formeln wiederholte und nach Nennung des genauen Datums und Ausstellungsortes unterzeichnete. Es folgen die insgesamt sieben (!) Zeugen, die nicht nur in eigener Handschrift ihre Namen nannten, sondern daneben in einem Beisatz in unterschiedlicher Ausführlichkeit die Bitte des Polheim zur Bezeugung, die Bestätigung des genannten Orts und der Zeit oder die Bitte zur Anbringung der eigenen Handschrift und des angeborenen Siegels

\footnotetext{
${ }^{66}$ Kannibal, 2v.

${ }^{67}$ Vielleicht ist auch diese Rolle eines Sekretärs aus dem Ritterstand als für den Testator stellvertretender Unterzeichner der Grund der Aufbewahrung der Urkunde beim Landmarschallamt. Diese Schriftunkundigkeit, aber auch das Beglaubigen durch die Petschaft und nicht ein Siegel, sowie der Inhalt insgesamt sind wohl Belege für den nichtadeligen Stand des Partisan. ${ }^{68}$ Partisan, $3^{\mathrm{v}}$.
} 
erwähnten. ${ }^{69}$ Dafür wurde auf die Angabe von Zeugen in der Corroboratio selbst verzichtet, dort wurde lediglich summarisch auf diese und die Bitte zur Anbringung von Siegel und Unterschrift verwiesen. ${ }^{70}$

Verwiesen sei auch auf mögliche Beilagen und nachträgliche Änderungen/Erweiterungen des Testaments: Neben den schon erwähnten zusätzlichen Schriftstücken bei Barbara Pösing legte auch Casimir zu Polheim seinem letzten Willen ein Inventar bei. ${ }^{71}$ Zudem bestätigte er dessen Rechtswirksamkeit sowie die von anderen beigelegten, eigenhändig verfassten Zettel als ob diese im Testamentstext eingeschrieben wären, auch wenn dadurch die ursprünglichen Verfügungen abgeändert würden. Solche Änderungen behielt er sich zusätzlich vor. ${ }^{72}$

Abschließend noch eine Bemerkung zu den zwei Pergamenturkunden: Diese wurden von Kanzleihand verfasst, Eufemia von Stubenberg und Wolf Streun zu Schwarzenau unterschrieben aber beide eigenhändig unterhalb des Textes, was nach Anbringung der Siegel von der Plica verdeckt wurde. Während Wolf Streun zu Schwarzenau lediglich seinen Namen und den Vermerk beifügte, dass diese Unterzeichnung in eigener Handschrift erfolge, steht nach der Nennung des Namens Eufemia, wobei zuerst deren Mädchenname Stubenberg, dann ihr Stand als Frau von Polheim folgte, eine kurze, schwer lesbare, weil in zittriger Handschrift verfasste Stellungnahme.

Insgesamt wird deutlich, dass die Fähigkeit zur eigenhändigen Unterfertigung in dem untersuchten Milieu eher Regel als Ausnahme war. ${ }^{73}$

\footnotetext{
${ }^{69}$ Polheim, $8^{\mathrm{r}}-9^{\mathrm{r}}$.

${ }^{70}$ Polheim, 7v.

${ }^{71}$ Polheim, $3^{\mathrm{v}}-4^{\mathrm{r}}$. Allgemein zur Anlage eines Inventars, auch zur Absicherung vor möglichen Ansprüchen, vgl. WESENER, Erbrecht 28f.

${ }^{72}$ Polheim, $5^{\mathrm{r}}-6^{\mathrm{v}}$.

${ }^{73}$ Ein besonders markantes, bereits erwähntes Beispiel von Illiteralität ist Jany Partisan, der aber wiederum
}

Im Fall der Apollonia Perner liegt wahrscheinlich die Abschrift eines eigenhändig verfassten Schriftstückes vor. Casimir zu Polheim verfasste eine umfangreiche eigenhändige Stellungnahme am Schluss seines Testaments und kürzere Angaben erscheinen etwa bei Eufemia von Stubenberg oder Thomas Ott zu Pierbaum. Somit war bei der untersuchten Schicht Schriftlichkeit gegeben. In manchen Testamenten wurde auch auf die Kontrolle des Inhalts des letzten Willens verwiesen: So vermerkte Casimir zu Polheim die von ihm getroffenen Vorgaben und das fleißige Überlesen des Stücks. ${ }^{74}$ Zelking etwa schrieb, dass sie ihren Zeugen das Testament selbst vorgelesen habe. ${ }^{75}$ Barbara Pösing hatte auf lateinisch korrespondiert. ${ }^{76}$

\section{Struktur}

Grundsätzlich ist vor der Herausarbeitung einer allgemeinen Struktur der Testamente, sofern eine solche nachgewiesen werden kann, festzuhalten, dass die einzelnen Formeln in ihrer Ausführlichkeit stark variieren, so charakterisiert etwa das Testament Casimir zu Polheims eine längere Ausformulierung der einzelnen Bestandteile. Das Formular weist in groben Zügen charakteristische Merkmale auf, wobei es starre und weniger starre Formeln gibt, auch die Anordnung innerhalb des Textes lässt Gemeinsamkeiten erkennen. Um das Auftreten der einzelnen Wendungen untersuchen zu können, wurden diese klassifiziert und deren Anordnung im Text tabellarisch erfasst. Diese Reihenfolge der einzelnen Bausteine ist nicht starr, aber grob eingehalten worden. Auffällig ist, dass die Frauentestamente dem folgenden Schema, v.a. im Bereich der Legate, besser entsprechen. Insge-

weder dem Herren- noch dem Ritterstand zugewiesen werden kann.

${ }^{74}$ Polheim, 8 r.

${ }^{75}$ Zelking, $4^{\mathrm{v}}$.

76 WERTNER, Grafen 233. 
samt lehrt etwa der letzte Wille des Hans von Görtschach, dass die im Folgenden vorgeschlagene Reihenfolge innerhalb der Legate nicht immer eingehalten wurde. Die Anfangs- und Endpassagen, also grob alles außer die Legate, lassen aber doch eine gewisse Reihenfolge erkennen, wobei einzelne Verschiebungen zu beobachten sind. Ein Vergleich der einzelnen Formeln nach ihrem genauen Wortlaut und damit eine Analyse möglicher Abhängigkeiten kann aufgrund des vorgegebenen Umfangs dieser Arbeit nicht gegeben werden, doch soll der Aufbau deutlich gemacht werden und mehr oder weniger charakteristische Beispiele, da es immer kleine oder größere Abweichungen gibt, zitiert werden.

Grundsätzlich ist das Testament in drei Bereiche $\mathrm{zu}$ teilen: In der einleitenden Passage erfolgt eine Invocatio und dann eine Begründung für die Abfassung des Testaments. Die Invocatio ist dabei relativ starr, beginnt regelmäßig mit „Im Namen der heiligen Dreifaltigkeit". Eine umfangreichere Variante bietet das Testament der Margaretha Prockh: „In dem namen der aller heiligisten, unzerthailten trivaltigkhait, Gott des vatters, Gott des shons und Gott des heiligen geists, unserer allgemainen christlichen anerkhennten waren ainigen Gothait, amen. ${ }^{47}$ Darin wird zusätzlich auf die allgemeine christliche Überzeugung des dreieinigen Gottes verwiesen. Wolf Streun zu Schwarzenau betonte bei der Nennung des Gottessohns und des heiligen Geistes, dass jener ihn erlöst und dieser ihm den Glauben an die Seligkeit offenbart habe. ${ }^{78}$ Es folgt in einigen Beispielen mit dem Verb „bekenne" die Publicatio (z.B. Eufemia zu Polheim, Thomas Ott zu Pierbaum, Wolf Streun zu Schwarzenau), dann der Name des Testators oder der Testatorin ${ }^{79}$ (Intitulatio) und allgemeine

\footnotetext{
77 Prockh, $1^{\text {r }}$.

${ }^{78}$ Schwarzenau.

${ }^{79} \mathrm{Vgl}$. etwa Polheim, $1^{\mathrm{r}}$ oder Pierbaum, $1^{\mathrm{r}}$, anders Schwarzenau („Habe ich $\left.[\ldots]^{\prime \prime}\right)$, so auch Kannibal, $1^{\mathrm{r}}$.
}

Gedanken, die die Einstellung zum Tod und der Welt, die oft als Jammertal bezeichnet wurde, widerspiegeln, die Sicherheit des Todes (Nihilcertius-morte-Formel) ansprechen ${ }^{80}$ und die moralische Verpflichtung zur Abfassung eines letzten Willens, um künftigen Streit zu vermeiden. ${ }^{81}$ Diese Formeln enthalten im Laufe dieser Gedanken den Verweis, dass aus den angeführten Gründen der letzte Wille aufgesetzt worden sei . Sofern diese etwas ausführlicher gestaltet sind, können daraus Aufschlüsse zur Glaubensüberzeugung der Testatoren gewonnen werden. Hingewiesen sei an dieser Stelle auf das bereits mehrfach erwähnte Testament des Casimir zu Polheim, das durch seine Ausführlichkeit besticht. ${ }^{82}$ Apollonia Perner hielt zur Motivation der Errichtung eines letzten Willens fest, dass in letzter Zeit viele unvorbereitet gestorben seien. ${ }^{83}$ Schwarzenau betonte, dass viele die Abfassung eines letzten Willens zur rechten Zeit verabsäumen würden und ein Testament aufgrund von Krankheit, damit einhergehender geistiger Beeinträchtigung oder plötzlichem Tod ausbliebe. ${ }^{84}$ Dann können bereits konkretere Angaben zur eigentlichen Abfassungsursache, etwa Krankheit oder Schwangerschaft, folgen. Auf diese Formeln folgt dann bereits der erste Abschnitt rechtserheblicherer Natur: Eingeleitet wird er

Besonders ausführlich mit Nennung des Ehemannes, dessen Funktion und den Namen der Eltern, bei der Mutter sogar jenen deren Vaters, erscheint die Intitulatio bei Catharina Steghofer (Steghofer, $1^{\mathrm{r}}$ ).

${ }^{80}$ Vgl. dazu ZAJIC, Gedächtnis 310f. und zur Vergänglichkeit in Grabinschriften allgemein 304-311. Als interessantes Missgeschick sei auf die verkehrte Formulierung im Testament des Partisan verwiesen, also, dass es nichts sicheres gäbe als die Stunde des Lebens und nichts unsicheres als die des Todes (Partisan, $1^{\mathrm{r}}$ ).

${ }^{81}$ Kannibal, $1^{\mathrm{r}}$ : ,[...] in den zeitlichen guettern, damit mih Gott der almechtig begabt hatt, auf das drumen nach meinem tödtlichen abgang, unainikhait, khrieg, irrung, zwitracht und aller misverstandt vermiden bleiben." Vgl. u.a. auch Irnfried, $1^{r}$.

82 Polheim, $1^{\text {r-v }}$.

${ }^{83}$ Perner, $1^{\mathrm{r}}$.

${ }^{84}$ Schwarzenau. 
zumeist mit der Sana-mente-Formel, wo auch ein Hinweis auf eine mögliche Krankheit gegeben werden konnte. ${ }^{85}$ Daran schloss sich eine Passage an, die zusätzlich die Geschäftsfähigkeit betonte, also die Möglichkeit zum Abfassen eines letzten Willens. ${ }^{86}$ Nicht immer, aber in mehreren Fällen dieser Auswahluntersuchung belegt, erfolgte nun eine zusätzliche Sicherung der Rechtsgültigkeit, indem festgehalten wurde, dass bei irgendeinem Mangel das Schriftstück zumindest als Verfügung auf niedrigerer juristischer Ebene, etwa als Kodizill, Bestand haben sollte. ${ }^{87}$ Diese Formel (Kodizillarformel, favor testamenti) ist fast immer am Schluss der Testamente zu finden, in wenigen Fällen eben auch zu Beginn. ${ }^{88}$ Es kann zusätzlich oder auch alleine die Betonung der Abfassung des Testaments in der allerbesten, also beständigsten Form einer festgelegten Rechtsnorm erfolgen. ${ }^{89}$ Dabei wird

${ }^{85} \mathrm{Nagl}, 1^{\mathrm{r}-\mathrm{v}}:$,"[...] bey gesundtem leib, guetter $\left[1^{\mathrm{v}}\right]$ vernunfft, frey willig, unbezwungen und unangelernt mit guettem gewißn, zeitlicher vorbedrachtung [...]"; Kannibal, $1^{\mathrm{r}}$ : „Demnach hab ich mit guettem freyem willen, zeitlicher betrachtung und erwegung, wiewol schwaches leibs aber bei zimlicher vernufft, $\mathrm{zu}$ der zeit, da ich das an menikhlichs widersprechen wol zuthuen macht gehabt habe, mein geschäfft, ordnung und lessten willen aufgericht und gemaht." Vgl. auch Prockh, 1'; Zelking, $1^{\mathrm{r}}$.

${ }^{86}$ Z.B. Zelking, 1 ${ }^{\mathrm{r}}$ : ,[...] zu der zeit ich das on meniglichs irrung, hindernuß und widersprechen thuen khundt $[\ldots]^{\prime \prime}$; vgl. auch Kannibal, $1^{\mathrm{r}}$ (s. oben nach Sana-mente-Formel); allg. OGRIS, Geschäftsfähigkeit 1594-1596; OGRIS, Testament 160f. Zur Testierfähigkeit s. auch WESENER, Erbrecht 122-126.

${ }^{87}$ So bei Irnfried, $1^{\text {r: }}$, ,[A]llso who dasselbig auß rechtmessiger zierligkhaitt mangl hette, daz doch die crafft unnd wierkhung des codicill unnd aines jeden lesten willens halben und für vollkhumentlich und unverändert gehallten unnd von aller obbrigkhait darfür erkhundt unnd gehandthabt werden solle“. Vgl. auch Stubenberg.

${ }^{88}$ Dieser favor testamenti stammt aus dem römischen Recht, OGRIS, Testament 163. Häufig ist eine Kombination aus Kodizillarklausel und anderer Testamentsformen (z.B. Görtschach, $5^{\mathrm{r}-\mathrm{v}}$ ).

${ }^{89}$ Zelking, 1v: „,[...] Mach, orden und beschleuß mein testament auch hiemit in besster formb, masß und in einigen Fällen zusätzlich angeführt, dass dies deshalb geschehe, damit das Testament vor geistlichem und weltlichem Recht, Richtern und Gerichten Bestand habe, auch jenen des Landes. ${ }^{90}$ Bei oder vor der Formel zur Rechtssicherung oder der Rechtsnorm kann beiläufig auch der Zeitpunkt des Inkrafttretens, nämlich der Tod des Erblassers oder der Erblasserin, erwähnt werden, der aber auch am Ende dieses Abschnitts ${ }^{91}$ oder überhaupt des letzten Willens (s. auch unten) ${ }^{92}$ stehen kann. Diese Erwähnung ist, vor allem wenn sie im ersten Abschnitt des Testaments erscheint, unterschiedlich positioniert. ${ }^{93}$ Schließlich kann bereits an dieser Stelle die Betonung des Widerrufsrechts erfolgen, also die Betonung der Möglichkeit zur Abänderung oder sogar Neufassung des Testaments. Im Fall der Barbara Pösing schloss daran die ausdrückliche Aufhebung eines früheren Testaments, was bereits erwähnt wurde. ${ }^{94}$ Bei Casimir zu Polheim erfolgte dies bei den Schlussverfügungen. ${ }^{95}$ Im zweiten Abschnitt folgen nun die einzelnen Verfügungen, die häufig mit einer Bitte an den barmherzigen Gott zur Gnade und Aufnahme der Seele in den Himmel beginnen (commendatio

gestalt, wie dasselbig vermüg der rechten und sonnderlich dem lanndtsbrauch nach in Osterreich under der Ennß am chrefftigisten und beständigisten imer sein khan oder mag." Vgl. auch Nagl, 1v (Österreich unter der Enns).

${ }^{90} \mathrm{Vgl}$. Stubenberg (s. oben).

${ }^{1}$ Zelking, $1^{\mathrm{v}}$ und auch schon vor Formel zur Verhinderung von Streit: ebd., $1^{\mathrm{r}}$.

92 So bei Görtschach, 5v. Dieses Inkrafttreten nach dem Tod steht bei Elisabeth Nagl nach den Verfügungen zur Aufteilung des Erbes und vor dem Widerrufsrecht. Vor dem Widerrufsrecht erscheint es auch im letzten Willen der Barbara Pösing (Pösing, 14²).

${ }^{93}$ Bei Catharina Steghofer erfolgt dies nach der Sanamente-Formel (Steghofer, 1 ${ }^{\mathrm{v}}$, auch bei Irnfried, 1 ${ }^{\mathrm{r}}$; bei Nagl vor der Sana-mente-Formel, 1 ${ }^{\mathrm{r}}$ ), bei Margaretha Prockh steht diese vor der Inkraftsetzung und Formel zur bestmöglichen Form (Prockh, 1v). Vgl. auch Zelking, $1^{\mathrm{r}}$.

${ }^{94}$ Pösing, $1^{\mathrm{v}}$.

${ }^{95}$ Polheim, $6^{\mathrm{v}}-7^{\mathrm{r}}$. 
animae). Der zweite Punkt beschäftigt sich in unterschiedlicher Ausführlichkeit mit der Bestattung. Häufig wurden dieses Befehlen der Seele in die Gnade sowie Barmherzigkeit Gottes und die Regelungen für das Begräbnis in einer Verfügung zusammengefasst.96 Geldspenden oder die Verteilung von bei der Bestattung verwendeten schwarzen Tüchern leiten über zu den Legaten, deren Struktur Gemeinsamkeiten erkennen lässt: Am Beginn werden die Armen, Spitäler und in einem Fall auch eine Kirche bedacht, ${ }^{97}$ es folgen in der Regel die nahen Verwandten, Freunde und Diener. An dieser Stelle oder am Ende der Verfügungen konnte eine allgemeine Abfertigung der anderen Verwandten erfolgen. ${ }^{98}$ Am Ende der Legate erscheint nun wieder häufig die nahe Familie, zumeist im Zuge der Erbeinsetzung und damit verbundenen Schuldentilgung. ${ }^{99}$ Dann wurden häufig die

\footnotetext{
${ }^{96}$ So u.a. bei Zelking, $1^{\mathrm{v}}$; Irnfried, $1^{\mathrm{r}-\mathrm{v}} ; \mathrm{Nagl}, 1^{\mathrm{v}}-2^{\mathrm{r}}$.

${ }^{97}$ Prockh, 2v (Stephansdom, gleichzeitig gewünschte Grablege).

${ }^{98}$ Vgl. auch Partisan, 3r: "Verrer schaff ich meinen negsten bluetsfreundten, so sich genuegsamb $\mathrm{zu}$ mir legitimiern zwainzig gulden reinisch $\mathrm{zu}$ funffzig pazen oder sechzig khreuzer zeraitten, damit sollen sy gennzlich von meinem guett abgeferttigt sein."; Pösing, 5 $5^{\mathrm{v}}-6^{\mathrm{r}}$ (50 Gulden; zwischen Legaten an Verwandte und andere). Diese betonte auch bei dem Legat an die Kinder ihres verstorbenen Schwagers, dass diese, da die Testatorin durch ihren Mann nichts ererbt, sondern im Gegenteil diesen und seine Verwandten mit Geld unterstützt habe, damit begnügt sein sollten (Pösing, $5^{r-v}$ ). Verwandtschaft ist mit dem Begriff der Freundschaft gleichzusetzen, vgl. u.a. BASTL, Tugend 38f.; GRIMM, Wörterbuch 4, 168. Catharina Steghofer bedachte ihre Verwwandten mit 100 Pfund Pfennig, die über ein Jahr nach ihrem Tod ausbezahlt werden sollten, falls sie ohne Kinder versterben sollte (Steghofer, 2v ). Zu Möglichkeit der allgemeinen Abfertigung mittels Klausel bzw. Noterbrecht kompakt OGRIS, Noterben 1056-1059. Zur Erbfolge allg. LIPP, Erbfolgeordnung 1361-1365. Zu Fallrecht LIPP, Erbfolgeordnung 1363f.; WESENER, Erbrecht 44-84, 170-186.

${ }^{99}$ Vgl. zur Haftung der Erben WeSENER, Erbrecht 2729.
}

Gerhaben mit ihren Pflichten bestimmt. ${ }^{100}$ Regina Zelking bestellte diese hingegen direkt nach den Verfügungen zu ihrer Bestattung. ${ }^{101}$ Es folgte eine Art Sanctio, in der festgelegt wurde, dass bei Unzufriedenheit und einem Einspruch gegen das Testament, die betreffenden Personen ihres Legats verlustig erklärt würden (Verwirkungsklausel102), manchmal auch der Zusatz, dass ansonsten das Testament bestehen bleiben solle. ${ }^{103}$ Margaretha Prockh übertrug die freien Legate ihrem Haupterben, nämlich ihrem Sohn, ${ }^{104}$ Barbara Pösing verteilte diese Beträge an die Spitäler in St. Marx, Pösing und Hainburg. ${ }^{105}$ Am Ende dieses Abschnitts konnte auch die Ernennung von Exekutoren mit der Festlegung deren Aufgaben erfolgen. ${ }^{106}$ Auf die Frage der Arten der Erbeinsetzung kann nur summarisch eingegangen werden, doch findet sich in der Regel die Benennung eines Haupterben oder dessen indirekte Einsetzung. Polheim: „[D]as furnemblichisst recht, wesentlich haubtstukh aines yeden tesstament oder lessten willens ist die institution, ernennung unnd sezung der erben.“107 Dadurch bestätigt er die Einschätzung

\footnotetext{
${ }^{100}$ Z.B. Stubenberg, Verf. 8; Görtschach, $4^{\mathrm{r}-\mathrm{v}}$; Schwarzenau.

101 Zelking, $1^{\mathrm{v}}-3^{\mathrm{r}}$.

102 OGRIS, Testament 162.

${ }^{103}$ Prockh, 8v: „Hierüber ist auch mein gänzlicher will und maynung, wo sich jemands meiner erben und befreundtn diesem meinem testament und lezten willen $\mathrm{zu}$ widerstreben, desselben inhalt $\mathrm{zu}$ gegen ichtes fürzunemen und zu hanndlen underfienge, das alspald desselben legat solle gefallen und verworcht sein, meinem sohn inhendig bleiben und dennocht nicht minder diß mein testament bey denen andern in sich haltunden articln, seinen wirden und crefften ungeschwecht gehalten und volzogen werden." Pösing, 6 ; ; Schwarzenau.

${ }^{104}$ Prockh, $8^{v}$, siehe vorige Anm.

105 Pösing, 6r.

106 Z.B. Steghofer, $3^{\mathrm{v}}-4^{\mathrm{r}}$; Irnfried, $4^{\mathrm{r}}$.

107 Polheim, 3r; zu dieser an das römische Recht angelehnten Formulierung vgl. OGRIS, Testament 153. 157; SEDATIS, Universalsukzession 489-492; WESENER, Erbrecht 119-121.
} 
Weseners, dass sich die Erbeinsetzung im 16. Jahrhundert durchsetzte. ${ }^{108}$

Beinahe in allen Testamenten befand sich nach diesem Block die Bitte an die Obrigkeit, häufig an den Kaiser selbst und/oder an die nachgesetzten Gewaltträger gerichtet, die Durchführung des letzten Willens zu überwachen und dieses vor aller Widerrede zu schützen. ${ }^{109}$ Dafür wurde für diese Glück, Segen und Wohlfahrt erbeten. ${ }^{110}$ Blieb diese Bitte zumeist anonym, nannte Apollonia Perner Kaiser Maximilian II. namentlich. ${ }^{111}$

Dann erfolgte erstmals oder wiederholend die Rechtssicherung bei äußeren Mängeln des Testaments, also, wie oben bereits und bei dem

108 WESENER, Erbrecht 192.

109 Stubenberg: „Hierauf ich unnderthenigist bitte die römisch khünigclich maiestet etc., meinen aller genedigisten herrn und lanndsfürsten, oder irer maiestat lanndshaubtman und nachgesezte obrigchait diß mein geschäfft und lessten willen hanndt zuhaben unnd gegen ainem yeden so das widerfechten, auch etwo durch personen undergetrugkht werden wollt, darob mit ernnst zehallten, damit disem gennzlichen voltzogen und nachgelebt werde." Polheim, 7 r: „Hirauf will ich auch die römisch khayserlich unnd khunigelich mayestat, meine aller genedigisste herrn unnd lanndsfürssten unnd all annder ordenlich hoch unnd nider obrigkhayt in gehorsamer, diemuettiger unnderthanigkhayt unnd alles muglichen geburlichen vleiß zum höchssten angerufft unnd gebetten haben; in massen ich sy dann allso aller vleissigisst unnd diemuettigist auch embsigisst ersueche, anrueff unnd bith, sy wellen durch Gott unnd in betrachtung, das inen alls eingesezten höchssten heubteren, obrigkhayten unnd magistratten, nach ordnung unnd aufsazung aller geistlicher und welltlicher rechten soliches innsonnderhait eingebunden ist, ob disem meinem alls aines vernufftigen menschen tesstamennt unnd ordnung lesstes willens mit rechtem ernnsst, allergenedigisst, genedig unnd gunstigelich hallten, damit nemblich dasselbe bey crefften, wierden sovil muglich bleibe unnd ungewaigert stragkhs volzogen werde, wie sy dann solches in iren aignen gewissen unnd in khunfftiger wellt vor den augen Gott unnsers himlischen vatters zuver anntwortten gedenkhen."

110 Pösing, 14 ${ }^{\mathrm{r}}$; Pierbaum, 3 ;

${ }^{111}$ Perner, $7^{\mathrm{r}-\mathrm{v}}$.
Kapitel zur Selbstbezeichnung der Dokumente dargestellt, dass es trotz solcher als mindere Form Bestand haben sollte. ${ }^{112}$ Wäre der letzte Wille, so Eufemia Stubenberg, nach dem gemeinen Recht nicht in ausreichender Form verfasst, sollte man sich doch danach richten, als ob das Testament nach den kaiserlichen, königlichen, geistlichen oder weltlichen Rechten, Statuten und Privilegien verfasst worden wäre und es demnach in der bestmöglichen Form vollziehen werden. ${ }^{113}$ Nach dieser Sicherung des Inhalts erschien dann häufig die Formel des Widerrufsrechts, dann folgte in der Regel das Inkraftsetzen des letzten Willens. ${ }^{114}$ Zur Formelhaf-

112 Polheim, 6 $6^{\mathrm{r}}$ (s. oben); Prockh, 9r: „Und ob dann auch nunmer über soliches alles bey diser meiner testamentlichen verordnung an derselben aigentlichen volkhomeren solemnitet und würckhung aines ordenlichen recht und lanndtsgepreüchigen zierlichen, crefftigen testaments ainicherlay manngl erschine und deshalben angefochten werden solte oder möchte, so will ich hiemit allen und jeden solichen manngl und abganng volkhomentlich erholt und erstatt haben. Seze, ordne und will auch, das solich mein testamentliche verordnung zum menigist, doch als ain testament vom mundt ausgesprochen, codicil, gab von todts wegen oder sonnst als ain gemaine freye wülckhürliche anordnung und beschliessung aines jeden bevorsteunden letzten willens, gelte, fueg, crafft und würckhung habe, darfür verstanden, gehalten, angenomen und volzogen werde."

${ }^{113}$ Stubenberg.

114 So etwa Stubenberg (Widerrufsrecht, dann Inkraftsetzen); Görtschach, 5v; Kannibal, 2r: „Ich gedahte Anna Khaniwallin wil mir auch auf diz mein ordnung, lezten willen und geschäfft hierinen nihts vorbehalten, allain, wo aus rechten beweglihen ursachen und furvalender noth mir was gelegen oder für guet ansehen wurdt, diz mein geschäfft in der zeit zumindern, mern oder gar von neuem aufzurihten. Wo das aber nit beschach in meinem leben, so soll es gantzlich bey disen meinem lezten willen und geschäfft bleiben und one menikhlih einredt und widertreiben, treilich und ongeverde nahgangen werden." Prockh, 10r: "Jedoch behalte ich mir hieneben auch bevor aller meiner fürfallenden gelegenhait und gefallen nach diß mein testament zuverändern, zu mindern, zu meren und gar zuverkhern, da aber hierüber khünfftigclich khain anders jungers und neuers durch mich 
tigkeit des Widerrufsrechts sei angemerkt, dass selbst bzw. gerade die schwerkranken Margaretha Prockh und Regina Zelking nicht auf diesen Passus verzichteten. ${ }^{115}$ Das Widerrufsrecht konnte aber auch zu Beginn des Blockes, etwa nach den Legaten und der Einsetzung der Gerhaben, stehen. ${ }^{116}$ Das Testament des Hans von Görtschach stellt insofern einen Sonderfall dar, als es das Inkraftsetzen und die Bitte an die höheren Stellen zur Wahrung des letzten Willens nochmals abschließend wiederholte, um dann die Wirksamkeit des Testaments erst ab dem Zeitpunkt des Todes zu betonen. ${ }^{117}$

Es folgt der dritte Abschnitt mit der Corroboratio, in der zur Bekräftigung des Dokuments der Name des Testators bzw. der Testatorin, die Art der Beglaubigung und, falls vorhanden, die Zeugen samt deren Unterfertigungsart mit Schadlosformel genannt wurden. Diese Angaben verschmolzen mit der Angabe des Ausstellungsortes und der Datierung. Unterschriften und Petschaften schlossen dann das Testament $\mathrm{ab}$. Betrachtet man die Ausstellungsorte, so fehlt die Angabe eines solchen nur bei Anna Kannibal. Ansonsten gilt es festzuhalten, dass der letzte Wille in der Regel im Herrschafts- bzw. Lebensmittelpunkt aufgesetzt wurde. Eufemia und Casimir zu Polheim testierten in Puchheim, Hans von Görtschach in Gross, Apollonia Perner in dem nach der Einigung in einem Erbschaftsstreit ihr verbliebenen Gut St. Johannes am Steinfeld, Mang Irnfried im Rottenhof, Elisabeth Nagl in Grafenegg, wo ihr Mann Pfleger war, Thomas Ott zu Pierbaum in Pierbaum und Wolf Streun zu Schwarzenau in eben diesem Schloss. Catharina Steghofer setzte ihren letzten Willen

aufgerichtet, befunden und fürkhomen würde, so will ich, das es alsdann bey disem gegenwürttigen meinem geschäfft gänzlichen also verbleiben solle." $S$. auch Nagl, $5^{\mathrm{r}-\mathrm{v}}$; Zelking, $5^{\mathrm{r}}$; Pösing, $14^{\mathrm{r}}$.

115 Pernfuss, $10^{\mathrm{r}}$; Zelking, $4^{\mathrm{v}}$.

${ }^{116}$ Steghofer, $4^{\mathrm{r}}$; Pierbaum, $3^{\mathrm{r}-\mathrm{v}}$.

117 Görtschach, $5^{\mathrm{r}-\mathrm{v}}$. in Stein auf, wobei sie ihre Zeugen aus dem inneren Rat daselbst schöpfte. Margaretha Prockh testierte in Wien, was durch ihre Ehe mit Hans Prockh erklärt werden kann, der auch Wiener Bürger war. Trotz oder gerade wegen dieser Verbindung bestand sie auf der Zuständigkeit des Landmarschalls bei ihrem letzten Willen (s. unten). Ebenso in Wien testierte Barbara Pösing. Letztere hatte in der Stadt ihren Wohnsitz. Auch Regina Zelking verfasste ihren letzten Willen dort. Festzuhalten ist auch die Rolle des Landuntermarschalls als Exekutor oder die Benennung eines in Wien lebenden, „wohlgelehrten“ Zeugen. Dabei mag es sich aber weniger um die Ursache bzw. den Anlass als eine Folge des Abfassungsortes handeln, umso verwirrender ist zudem, dass Zelking Häuser in Znaim und Korneuburg besaß, in letzterer Stadt auch bestattet werden wollte. Auch Jany Partisan setzte in Wien seinen letzten Willen auf, wobei dies durch dessen das Haupterbgut darstellenden Besitz bei den Minoriten erklärbar ist. ${ }^{118}$

Zusammenfassend sei angemerkt, dass auf bestimmte Formeln in keinem oder nur wenigen der untersuchten Dokumente verzichtet wurde: In keinem Fall fehlt die Sana-mente-Formel. Allein Casimir zu Polheim und Mang Irnfried verzichteten auf den Hinweis auf die Geschäftsfähigkeit; konkret die Formel, dass man den letzten Willen verfasste "zw der zeit, das ichs an aller menigkhlichs rechtlichs widerspröchen “119 konnte. Eine rechtssichernde Formel oder Kodizillarformel ist bis auf den letzten Willen der Anna Kannibal immer vorhanden, zumeist am Beginn und Ende des Dokumentes. Das Widerrufsrecht fehlt allein bei Jany Partisan. Bereits erwähnt wurde, dass allein Anna Kannibal auf die Benennung von Zeugen und die damit zusammenhängende Schadlosformel verzichtete.

\footnotetext{
${ }^{118}$ Vgl. zu den Angaben die Tabelle.

${ }^{119}$ Görtschach, $1^{\text {r }}$.
} 
Auch die Bitte an die Obrigkeit konnte fehlen, nämlich bei Catharina Steghofer, Mang Irnfried, Wolf Streun zu Schwarzenau oder Jany Partisan. Abgesehen von diesen für die Rechtssicherheit interessanten Passagen kann noch darauf verwiesen werden, dass in allen Testamenten bis auf den letzten Willen von Mang Irnfried eine Nihil-certius-morte-Formel vorhanden ist, häufig wird auch auf die Einstellung zum Tod als natürlich bzw. angeborene menschliche Schuld und das Jammertal bzw. elende Leben auf der Welt verwiesen. Letzterer Verweis fehlt allein bei Hans von Görtschach, Apollonia Perner und Regina Zelking. Immer wird die Seele in die Barmherzigkeit oder Gnade Gottes empfohlen und Anweisungen zur Bestattung gegeben. Diese zusammenfassende Aufzählung soll noch einmal die Gemeinsamkeiten verdeutlichen, zeigt aber, dass manche Testamente, wie jenes von Mang Irnfried, durchaus in mehreren Punkten aus dem beobachteten Rahmen fallen konnten.

Bei den erhaltenen Ausfertigungen befindet sich ein Rubrum mit Angabe des Inhalts auf der Rückseite des Dokumentes oder, wenn vorhanden, am Kuvert. Diese kurzen Angaben sind insofern bedeutend, da sie Zusatzinformationen zur Eröffnung oder im Umgang mit dem Schriftstück enthalten können.

\section{Hinweise zur Abfassung, Beglaubigung und Vollstreckung}

Die anzuwendende Rechtsnorm wurde in einigen Testamenten explizit genannt, dabei in der Regel auf das Recht im Land unter der Enns verwiesen. ${ }^{120}$ Barbara Pösing legte als einschlägiges Recht in ihrem Testament von 1568 das der Länder Österreich ob und unter der Enns fest. $^{121}$ In dem Kodizill von 1571 verwies sie

${ }^{120}$ U.a. Steghofer, 1v; Schwarzenau.

${ }^{121}$ Pösing, $1^{\mathrm{r}}$. noch einmal darauf, dass sie in den österreichischen Landen wohnhaft war und nichts von ihren Eltern im Königreich Ungarn erhalten habe. Aufgrund des eigenständigen Erwerbes ihres nunmehrigen Besitzes, der damit verbundenen Verfügungsfreiheit und weil sie in Österreich wohnhaft sei, untersagte sie explizit eine Abhandlung nach dem Gebrauch des Königreichs Ungarn, falls Verwandte beiderlei Geschlechts dieses aufgrund ihrer Geburt ebendort einfordern würden. ${ }^{122}$ Damit versuchte sie in ihrem Kodizill mögliche Einsprüche gegen ihren letzten Willen ausdrücklich $\mathrm{zu}$ entkräften. Casimir zu Polheim und seine Frau Eufemia bezogen sich auf das Recht in Österreich ob der Enns. ${ }^{123}$

$\mathrm{Zu}$ den Vorgängen zur Entstehung und dann Durchführung der letzten Willen geben die Dokumente selbst nur vereinzelte Hinweise. Zum einen wurde in der Corroboratio stets auf die Art der Beglaubigung verwiesen, zum anderen gab es in wenigen Fällen den Verweis auf die Eröffnung des Testaments.

Als Grund für die Abfassung des Testaments wurde bei den untersuchten Beispielen in der Regel die von Gott auferlegte Pflicht eines Christen, seine Angelegenheiten zu ordnen, um künftigen Streit um das Erbe zu vermeiden, angegeben. Seltener war eine genauere Angabe zum unmittelbaren Grund: Im Testament der Elisabeth Nagl erwähnte man bei der Aufzählung der Legate auch deren Schwangerschaft. Das ungeborene Kind wurde dabei je nach Geschlecht im Fall des Erreichens der Vogtbarkeit

\footnotetext{
${ }^{122}$ Pösing, 4r, 15 ${ }^{\mathrm{r}-\mathrm{v}}$. Zur freien Verfügung über Kaufgut bereits im Mittelalter vgl. OGRIS, Testament 154. Pösing bietet somit auch ein Beispiel des vom römischen Recht herrührenden Anspruchs auf Testierfreiheit (HAGEMANN, Erbrecht 1377f.; OGRIS, Testament 157), wobei diese zudem betont, dass sie die Güter selbst erworben und von den eigenen Verwandten nichts geerbt habe (Pösing, $2^{\mathrm{r}}-4^{\mathrm{r}}$ ).

${ }^{123}$ Polheim, 1v; Stubenberg.
} 
unterschiedlich bedacht, ${ }^{124}$ was an die geschilderten Bestimmungen der später abgefassten Landtafel erinnert. Für Nagl lässt sich vermuten, dass die Schwangerschaft und die Gefahren bei der Geburt Anlass zur Abfassung des letzten Willen waren. Da sie schon mehrere Kinder zur Welt gebracht hatte, wären ältere Dokumente zu erwarten, doch wurde auf diese nicht verwiesen. Noch deutlicher ist der Grund bei Erwähnung einer Krankheit oder Leibesschwachheit, wie etwa bei Anna Kannibal,125 Margaretha Prockh'126 oder Regina von Zelking. ${ }^{127}$ Partisan meinte wiederum, dass er das Testament auch in Anbetracht seines Alters aufgesetzt habe. ${ }^{128}$ Auf dieses verwies auch Barbara Pösing in ihrem letzten Kodizill von 1574 (,wie des lieben alters arth, so mit mancherley nach göttlichen willen beschwerd ist"). ${ }^{129}$ In relativ jungen Jahren verfasste Wolf Streun zu Schwarzenau sein Testament, der erst über 30 Jahre nach Aufsetzen des Testaments verstarb. Ansonsten liegt maximal ein Jahrzehnt zwischen Abfassen und Tod, bei den bereits erwähnten unmittelbaren Anlässen konnte der Tod relativ unmittelbar auf diese folgen (vgl. Tabelle).

Der Akt der Testamentsabfassung, also wer die Formulierung der Geschäfte und ihre Ausstellung vornahm, kann allein aus den Dokumenten kaum erschlossen werden. Auch ob dies am Ausstellungsort geschah oder nicht, muss offen bleiben. Einzig im Testament von Wolf Streun zu Schwarzenau erhält man einen Hinweis: Dieser belohnte seinen Untertanen, der zum Zeitpunkt der Testamentserrichtung in Vitis (Gerichtsbezirk Waidhofen an der Thaya) ${ }^{130}$ als

\footnotetext{
${ }^{124} \mathrm{Nagl}, 3^{\mathrm{v}}$.

${ }^{125} \mathrm{Kannibal}, 1^{\mathrm{r}}$; an anderer Stelle verweist sie auch auf die Langwierigkeit ihrer Krankheit (Kannibal, 2r).

126 Prockh, $1^{\mathrm{r}-\mathrm{v}}$.

127 Zelking, $1^{\text {r }}$.

${ }^{128}$ Partisan, $1^{\mathrm{r}}$.

129 Pösing, 19r.

${ }^{130}$ Die Visitation von 1540 ergab, dass der Pfarrer dort den Gottesdienst nach altem Gebrauch verrichtete.
}

Schulmeister tätig war, mit einem Betrag von 50 rheinischen Gulden, wobei er die „schreibung“ des Testaments als explizite Leistung auswies. ${ }^{131}$ Damit wird wahrscheinlich die tatsächliche Verfertigung, aber vermutlich auch eine Hilfestellung bei der Formulierung selbst gemeint sein. Entscheidend ist, dass dadurch zumindest an einer Stelle die Schreiberschicht eines solchen Testaments fassbar wird, die Tatsache, dass die betreffende Person Schulmeister ist, belegt den hohen Bildungsgrad dieser Leute im Hintergrund. Dieser war angesichts der formelhaften Wendungen aber auch notwendig, wie bei der Diskussion der einzelnen Testamentbausteine bereits deutlich wurde. Aufschlussreich sind auch die bereits erwähnten Unterfertigungen im Testament des Casimir zu Polheim: „Ich Casimier freyherr zw Polhaim unnd Wartenburg etc. bekhen hiemit das alles unnd jedes so meinenthalben in disem lybell begriffen, aus meinem sonndern bevelch und auff mein vleissigs uberlesen in diesen formb gepracht worden, weliches ich mit austrugkhlichen wordten, daneben auch durch dise mein selbs aigne handtschrift unnd hier anhangendem insigl, eröfnet unnd bekhenndt habe."132 Das Testament wurde von dem Schreiber also nach seinen Vorgaben und Wünschen verfasst, wobei wahrscheinlich dessen oder ein juristischer Rat eingeholt wurde, ${ }^{133}$ und

Auch wurde erwähnt, dass Wolf Streun diesem einen Zehent entzogen hatte, vgl. WIEDEMANN 1880, $618 \mathrm{f}$.

131 Schwarzenau.

132 Polheim, 8 .

133 Einschränkend dazu kann darauf verwiesen werden, dass sechs Jahre zuvor seine Frau Eufemia ihr Testament verfasst hatte und deshalb vielleicht auf die dabei gemachten Erfahrungen zurückgegriffen werden konnte, wobei dies eine Einbindung Casimirs oder einer seiner Vertrauten bei der Entstehung von deren Testament voraussetzt, wenn man nicht allein den fertigen Text als Vorgabe interpretieren will. Eine gewisse Übereinstimmung der beiden Dokumente wird einerseits durch die bereits erwähnte Verwendung von Fachtermini, andererseits etwa auch durch die gleiche, für die untersuchten Beispiele ungewöhn- 
durch ihn selbst Korrektur gelesen, was wiederum für den Bildungsgrad Polheims aufschlussreich ist.

Ebenso aufschlussreich ist die Beglaubigung des Testaments, also die Bestätigung des Inhalts durch Zeugen. Betrachtet man wiederum den gesetzlichen Rahmen, den die oberösterreichische Landtafel vorgab, dann wurden dort allgemein alle Personen, die auch in anderen Angelegenheiten zeugenfähig sein konnten, für Testamente zugelassen, wobei Frauen ausgeschlossen waren. ${ }^{134}$ Frauen konnten aber bei einem Kodizill als Zeugen auftreten. ${ }^{135}$ Ausgenommen waren auch die in dem letzten Willen eingesetzten Erben sowie deren Verwandte, was auch Schwiegerkinder bzw. Schwagerschaften zum Zeitpunkt der Abfassung des letzten Willens einschloss, wobei die Verfügung eines liegenden oder fahrenden Guts kein Ausschließungsgrund war, außer es würde einem Viertel der Verlassenschaft entsprechen. Personen, die selbst kein Testament errichten oder in keinem eingesetzt werden konnten, waren gleichfalls ausgeschlossen. ${ }^{136}$ Die Zahl der Zeugen variierte in den vorliegenden Stücken, ebenso auch die notwendigen Vorgaben der Landtafel. Eufemia von Stubenberg und Casimir zu Polheim benannten je sieben, allein Barbara Pösing griff auf die gleiche Zahl bei ihrem Haupttestament zurück. ${ }^{137}$ Vier erschienen bei Catharina Steghofer,

lich hohe Zeugenzahl von sieben Personen nahe gelegt.

134 Von dieser Regelung gab es insofern Ausnahmen, als Frauen im Fall gesundheitlicher oder anderer Notfälle durchaus als Zeugen akzeptiert wurden, STRÄTZ, Landtafel Titel 8, § 2, 326. Zur Zeugenfähigkeit zusammenfassend WESENER, Erbrecht 151-153.

135 STRÄTZ, Landtafel Titel 25, § 9, 362.

136 STRÄTZ, Landtafel Titel 11, 330f.

137 Sieben Zeugen forderte das römische Recht für einen mündlichen letzten Willen, was wiederum die Nähe der Testamente der Polheims zu diesem belegt (OGRIS, Testament 158). Dies wurde 1512 in der Reichsnotariatsordnung bestätigt (HAGEMANN, Erbrecht 1378; WESENER, Erbrecht 129). Damit wird auch lediglich zwei bei Mang Irnfried. Ansonsten wurden stets drei Zeugen benannt. ${ }^{138}$ In 91,7\% der von Bastl untersuchten Fälle für die zweite Hälfte des 16. Jahrhunderts erscheinen auch zwei bis drei Personen in dieser Funktion. ${ }^{139}$ Betrachtet man die vorliegenden Beispiele, wurden die gesetzlichen Vorgaben nur teilweise erfüllt: Frauen fehlen zwar, doch gibt es bei mehreren Zeugenlisten Verweise auf die Verwandtschaft der betreffenden Personen, so traten etwa mehrfach Schwäger auf, wobei es sich bei der entsprechenden Bestimmung zur Schwagerschaft um einen späteren Einschub bzw. eine Korrektur im Landtafeltext handelt. ${ }^{140}$ Wolf Schwarzenau ließ seinen Vetter Wolf von Hofkirchen seinen letzten Willen bezeugen. ${ }^{141}$ Die Zeugen konnten unter dem Text siegeln, unterschreiben oder das Stück durch ihre Siegel verschließen. Auffallend ist die Betonung dieser als ehrbare Leute, womit wohl deren Unbescholtenheit und damit Zeugenfähigkeit herausgestrichen werden sollte. ${ }^{142}$

Der nächste Punkt in der Landtafel legte eine bereits erwähnte Beobachtung fest, nämlich die Notwendigkeit der Aufforderung oder besser Bitte an die Zeugen mittels „Petzetl“. Es wurde

der Verweis bei der Kodizillarformel von Polheim besser verständlich, der ja neben einem Kodizill auch auf die mögliche Bewertung als mündliches Testament hinwies.

${ }^{138}$ Damit entsprechen die Stücke der für fremdhändige (allographe) Testamente beobachteten Praxis von zwischen zwei und sieben Zeugen (OGRIS, Testament 158) bzw. mit einer Ausnahme drei und sieben Zeugen (WeSENER, Erbrecht 133). Die Mindestzahl von zwei wird bereits im Neuen Testament (Matthäus 18, 19-20; Johannes 8, 17), aber auch im römischen Recht festgelegt (Digesten 22, 5, 12). Vgl. dazu FISCHER, Zeugen $1687 \mathrm{f}$.

${ }^{139}$ BASTL, Tugend, Liebe, Ehre 109. Zu dieser Zahl ist aber anzumerken, dass die Materialgrundlage von Bastl für die in dieser Arbeit untersuchte Zeit gering ist.

${ }^{140}$ Vgl. Görtschach, 5v-6r; Prockh, 10v .

${ }^{141}$ Schwarzenau.

142 OGRIS, Testament 159. 
bestimmt, dass bei einem schriftlichen Testament, wenn der Testator lesen und schreiben konnte, die Aufforderung der Zeugen über solche „Petzetl“ erfolgen musste. Diese mussten mit Tag und Jahr dem Datum des Testaments entsprechen sowie mit eigener Handschrift und Petschaft bestätigt werden. Konnte der Testator weder schreiben noch lesen, so musste er einen anderen bitten, neben ihm seine Petschaft aufzudrücken, konnte der Testator nur unterschreiben galt dasselbe. ${ }^{143}$ Bei Fehlen einer eigenen Unterfertigung eines illiteraten Testators musste laut Landtafel das Schriftstück durch zwei ehrliche Personen dem Zeugen überbracht werden oder dieser persönlich zum Testator kommen. Ansonsten ist eine gleichzeitige Beglaubigung aller Zeugen an einem Ort nicht notwendig. Bereits klar wurde, dass das Testament laut Landtafel neben den „Petzetln“ von einem zum anderen Zeugen gebracht werden konnte. Bei einem mündlichen letzten Willen reichte die mündliche Bitte, doch mussten alle Zeugen persönlich sowie gemeinsam die Verfügungen der Testatoren anhören. ${ }^{144}$ Immer wieder wurde in der Corroboratio der vorliegenden Stücke auf das fleißige Bitten durch den Testator bzw. der Testatorin, in drei Beispielen explizit auf Bittzettel („petzedln“) verwiesen. ${ }^{145}$ Diese Verweise geben keine genaueren Aufschlüsse zum Ablauf dieses Ersuchens, bei den Zeugenfertigungen des Casimir zu Polheim wiesen zwei auf den Erhalt der schriftlichen Bitte hin, so dass diese den betroffenen Personen zugeschickt wurde. Diese beiden, von denen einer Anwalt in Österreich ob der Enns war, bestätigten interessanterweise als einzige der insgesamt sieben Zeugen Ort, Tag und Stunde des Aktes. ${ }^{146}$ Zumindest die gleich-

143 WESENER, Erbrecht 135-139.

144 STRÄTZ, Landtafel Titel 12, 331-333.

145 Polheim, 8v-9r; Nagl, 6r; Pierbaum, 3v.

146 Polheim, 8v-9r. Damit wird auch die Vorgabe des Landesbrauchs zur Abgabe der Zeugenschaft an einem Tag erfüllt (WESENER, Erbrecht 138). zeitige Beglaubigung des Testaments von Testator und Zeugen ist in diesem Fall damit belegt, was nach den bereits erwähnten Vorgaben des jüngeren Landrechts nicht immer notwendig war. In den letzten Willen von Regina Zelking oder Elisabeth Nagl wurde auch explizit darauf verwiesen, dass die Zeugen den Inhalt angehört hatten. Bei ersterer wurde der Wille zuerst mündlich diesen mitgeteilt und dann unterfertigt, was an die Verfügung der Landtafel erinnert. Letztere hielt zudem fest, dass sie die Zeugen auch schriftlich mit Bittzetteln zur Beglaubigung gebeten hatte. ${ }^{147}$ Leider sind keine Beispiele dieser Schreiben bei den hier untersuchten Stücken erhalten, ${ }^{148}$ auch wenn diese laut Landtafel bei der Eröffnung offenbar vorgewiesen werden mussten. Das Vorlesen der Testamente wird etwa durch die Publicatio bei Casimir zu Polheim deutlich, die u.a. auch alle einschloss, denen es verlesen wurde. ${ }^{149}$ Bei Partisan trat neben die Zeugen eine weitere Person, die stellvertretend die Unterschrift für den schriftunkundigen Testator übernahm (s. oben).

Nicht unerwähnt soll in diesem Zusammenhang bleiben, dass einige Zeugen Funktionen in der Landesverwaltung oder in Städten innehatten, etwa Sekretäre im Land ob oder unter der Enns waren und damit durch persönliche Erfahrung und ihre Ausbildung vielleicht auch Einfluss auf die Formulierung des Testaments hatten, zumindest dessen formale Richtigkeit kontrollieren konnten und damit der Beglaubigung größeres Gewicht verliehen. ${ }^{150}$ Auch das für studierte

147 Zelking, 4v ; Nagl, 6r („müntlich von mir angehört“).

${ }^{148}$ Auf einen solchen aus dem Bestand des Niederösterreichsichen Landmarschallamts für das Jahr 1657 verweist WESENER, Erbrecht 137, Anm. 42.

149 Polheim, 1 r.

${ }^{150}$ Görtschach, $7 \mathrm{r}$ (Beisitzer der Landrechte im Land unter der Enns); Steghofer, $4^{v}$ (Sekretär und Landschreiber in Österreich unter der Enns, Ratsmitglied der Städte Krems und Stein); Prockh, 10 ${ }^{\mathrm{r}-\mathrm{v}}$ (Niederösterreichischer Kanzler, Kommissare der Niederösterreichischen Kanzlei); Zelking, 4v (Hofrichter zu Her- 
Personen charakteristische Würdeprädikat "hoch-“ oder „wohlgelehrt" scheint manchmal auf. ${ }^{151}$ Schließlich taucht bei manchen Testamenten ein Doktor der Rechte auf, etwa bei Barbara Pösing. ${ }^{152}$ Allgemein kann daher vermutet werden, dass die Testatoren auch bei Leuten aus ihrem Umfeld Rat einholten, so wird auch im letzten Willen von Hans von Görtschach ein Doktor beider Rechte, den er zusätzlich als Schwager bezeichnete, bedacht. 153

Nach der Abfassung des letzten Willens konnte eine gänzliche Neufassung oder teilweise Veränderung notwendig werden, beispielsweise durch den Tod eines der Begünstigten, wie etwa bei Barbara Pösing. ${ }^{154}$

An dieser Stelle seien für die vorliegenden Stücke kurz die in der Landtafel von Österreich ob der Enns angeführten Gründe für die Ungültigkeit eines Testaments aufgezählt: Jedem blieb es überlassen, sein Testament zu Lebzeiten zu ändern oder neu aufzusetzen, selbst wenn er ausdrücklich in seinem letzten Willen festhielt, dass er diesen nicht mehr abändern werde. Zur Aufhebung musste der Erblasser dieses allein vor der Obrigkeit oder drei ehrlichen, zeugenfähigen Personen widerrufen. Diese Ungültigkeitserklärung hatte selbst dann Gültigkeit, wenn kein anderes Testament mehr verfasst werden würde. Wurde ein ordentliches Testament auf-

zogenburg, Prokurator beim Landrecht in Österreich unter der Enns); Nagl, $6^{\mathrm{r}}$ (Schlüsselamtmann in Krems, zwei innere Räte der Stadt); Pösing, 14v (u.a. Landuntermarschall in Österreich unter der Enns, niederösterreichischer Kammerrat, niederösterreichischer Kammersekretär, Sekretär der Landschaft im Land unter der Enns) $18^{v}$ (Niederösterreichischer Kanzler der Regierung, Sekretär der Landschaft) $21^{\mathrm{v}}$ (Doktor der Rechte); Pierbaum, $3^{\mathrm{v}}-4^{\mathrm{r}}$ (Landuntermarschall in Österreich unter der Enns, Beisitzer der Landrechte); Partisan, 3v (Sekretär der Landschaft in Österreich unter der Enns).

151 Z.B. Prockh, 10 ${ }^{\mathrm{r}}$; vgl. auch Zelking, $4^{\mathrm{v}}$; Pösing, $14^{\mathrm{v}}$.

152 Pösing, 21 ${ }^{\mathrm{v}}$.

${ }^{153}$ Görtschach, $3^{\text {r }}$.

154 Pösing, 19r $-21^{\mathrm{v}}$. gesetzt, dieses nach einem Jahr aber durch ein neues ersetzt, ohne das erste förmlich aufzuheben, galt dieses ältere trotzdem als ungültig, außer wenn in diesem explizit erklärt wurde, dass kein jüngeres gelten sollte. Der jüngere letzte Wille musste auch in seiner Form dem Landesbrauch entsprechen, um gültig $\mathrm{zu}$ sein. Mit dem Hintergrund dieser Bestimmung ist die bereits erwähnte Formulierung bei Casimir zu Polheim zu verstehen, der festhielt, dass dieses Testament auch gültig bleiben sollte, wenn ein jüngeres auftauchen würde, außer bei expliziter Aufhebung des älteren letzten Willens, was somit den Vorgaben der Landtafel entsprach. ${ }^{155}$ Auch wenn Kinder oder Enkel geboren wurden, die im Testament nicht bedacht worden waren, dafür aber fremde Erben, war dieses nicht rechtmäßig. ${ }^{156}$ War die Mutter mit einem Kind schwanger, musste dieses wie die bereits geborenen bedacht werden. Geschah dies nicht, blieb das Testament auch ungültig, wenn das Kind bald nach der Geburt sterben sollte. Um dem vorzubeugen, wurde in der Landtafel eine Formulierung vorgegeben, die besagte, dass bei einem nachgeborenen Kind dieses $\mathrm{zu}$ gleichen Teilen als Miterbe benannt werde, falls es aber nicht lebendig zur Welt kam, der letzte Wille trotzdem Kraft haben sollte. Vor allem Sicherheitsklauseln betreffend künftiger Erben finden sich in den vorliegenden Testamenten häufig, wenn auch manchmal nur in Nebensätzen. ${ }^{157}$ Die untersuchten Testamente bieten außer dem Hinweis eines bereits ungültig gemachten Testaments bei Barbara Pösing dazu keine weiteren Anhaltspunkte.

\footnotetext{
155 Polheim, $6^{\mathrm{r}-\mathrm{v}}$.

${ }^{156}$ Zum Noterbenrecht s. oben, speziell dazu auch HAGEMANN, Erbrecht 1378f.

${ }^{157}$ Vgl. dazu etwa Verfügungen des zum Abfassungszeitpunkt des Testaments kinderlosen Wolf Streun zu Schwarzenau. Allg. STRÄTZ, Landtafel Titel 19, 348351. Vgl. zum Noterbrecht OGRIS, Noterben 10561059.
} 
Nach dem Tod des Erblassers oder der Erblasserin musste der nächste Schritt durch die Exekutoren, die Willensvollstrecker erfolgen. Allgemein wurden diese zur Durchführung oder zur Überwachung der der Intention des Testators oder der Testatorin entsprechenden Vollstreckung der Verfügungen eingesetzt. Aus den letzten Willen selbst erfährt man selten mehr als diese allgemeine Einsetzung, ${ }^{158}$ manchmal definierte der Testator bzw. die Testatorin die Aufgaben genauer. Ausführliche Angaben zum Umgang mit dem hinterlassenen Erbe verfasste Barbara Pösing, die den Exekutoren Anweisungen zur Anlage des Barvermögens, wobei sogar die Vorgabe des erhofften Zinsfußes von sieben Prozent nicht fehlte, und dessen Nutzung gab. So sollten diese alle ausständigen Ansprüche bei oder außerhalb eines Gerichts begleichen sowie Verfügungen für Waisen aus den jährlichen Zinsen vollziehen.159 Allein den Exekutoren sollte es vorbehalten sein, die einzelnen Legate entsprechend den Anordnungen auszugeben, wobei bereits durch Quittungen belegte Auszahlungen an die Begünstigten von der bestimmten Summe abzuziehen seien. Zudem wurde ihnen aufgetragen vorhandene Schulden, auch wenn diese auf andere Personen transferiert worden seien, einzutreiben. ${ }^{160}$ Mehrfach betonte die Testatorin, dass die Exekutoren auf die Unzertrennbarkeit der veranlagten Barvermögen achten sollten. ${ }^{161}$ Damit erscheinen sie als aktive Verwalter des Erbes. Insgesamt überschneiden sich die Aufgaben mit denen von bestellten Gerhaben bzw. wurden die Vollstrecker auch zu solchen ernannt. ${ }^{162}$ Selten fehlte die Bitte an die

158 Z.B. Stubenberg; Steghofer, $3^{\mathrm{v}}-4^{\mathrm{r}}$; Irnfried, $4^{\mathrm{r}}$.

${ }^{159}$ Pösing, 10v-11 ${ }^{\text {r }}$.

160 Pösing, 10v-13v

${ }^{161}$ Beispielhaft für die 16.000 Gulden an Oswald von Eitzing und seine Frau Barbara s. Pösing, 13ㄷ․

${ }^{162}$ Die Gerhaben werden an folgenden Stellen ernannt: Stubenberg, 8; Görtschach, $4^{\mathrm{r}-\mathrm{v}}$; Polheim, $3^{\mathrm{v}}-4^{\mathrm{v}}$; Steghofer, $2^{\mathrm{v}}-3^{\mathrm{r}}$ (nicht explizit als Gerhabe ernannt!); Prockh, $8^{\mathrm{r}}$ (=Exekutoren); Zelking, $1^{\mathrm{v}}-3^{\mathrm{r}}$; Irnfried, $4^{\mathrm{r}}$
Obrigkeit, das Testament anzuerkennen und den Verfügungen gemäß durchzuführen. ${ }^{163}$ Einen Sonderfall stellt eine Verfügung der Apollonia Perner bezüglich eines Gutes mit umstrittenen Besitzrechten dar, nämlich St. Johann am Steinfeld. Dieses war laut ihrem letzten Willen ihren Eltern von ihrem Schwiegervater Berthold Perner, dem Vater ihres Mannes Niklas, versetzt worden und dann in deren Eigentum übergegangen, wobei auf die Bitte Apollonias bei ihrer Mutter das Gut samt Nachlass des Versatzes darauf dem Perner überlassen blieb, aber die Besitzrechte an sie übergehen sollten. ${ }^{164}$ Dies hatte ihr auch ihr Mann zugesagt und am Totenbett verfügt. Dessen Testament bestätigte zudem, dass er von ihr das Geld zur Auslösung seiner Herrschaften erhalten hatte. ${ }^{165}$ War die Inbesitznahme der Burg Bärnegg durch Niklas Perner von Konflikten geprägt, so sollte seinem letzten Willen dasselbe Schicksal zu Teil werden: Diesen ließ Apollonia dessen Schwester sowie den Kindern, nämlich Michael Rindsmaul und einem Vertreter der Tochter, vorlesen, die dagegen sofort protestierten. Sogar die Möglichkeit einer Fälschung oder die Existenz von zwei Testamenten, da Niklas nicht lesen und schreiben konnte, Apollonia aber Siegel sowie Petschaft des Verstorbenen selbst aufbewahrt hatte, wurden erwogen. Den Konflikt trug man über die steirische Landesobrigkeit aus, der ehemaligen Universalerbin verblieben lediglich nach einem Vergleich eben St. Johann am Steinfeld, die Fahrhabe und ein Geldbetrag. ${ }^{166}$ Die Verfü-

(=Exekutoren); Pierbaum, 3r; Partisan, $3^{\mathrm{r}}$ (=Exekutoren). Vgl. allg. OGRIS, Testament 163f.; SCHMIDTWIEGAND, Vormund 1049f.; ERLER, Vormundschaft 1050-1055; WESENER, Erbrecht 187-190.

${ }^{163}$ Bei Fehlen wird diese Aufgabe den Exekutoren übertragen (z.B. Steghofer, $3^{\mathrm{v}}-4^{\mathrm{r}}$ ).

${ }^{164}$ Die Besitzstreitigkeiten fügen sich in einen größeren Konflikt um den Besitz der Perner von Bäreneck ein, dem hier nicht nachgegangen werden kann (PosCH, Hartberg 11-14).

165 Posch, Hartberg $12 f$.

166 POSCH, Hartberg 12-14. 
gung im Testament war ein Reflex dieses Prozesses: Darin erwähnte sie ihre alten Ansprüche auf das verbliebene Gut, auch die persönliche Anwesenheit des Michael Rindsmaul am Totenbett des Gatten, wo laut Apollonia dieser ihm aufgetragen hatte, ihr das Gut zu übergeben, und schließlich die persönliche Anwesenheit des Bruders Erasmus zur Unterstützung der Ansprüche seiner Schwester im Landhaus von Graz. Tatsächlich behauptete Rindsmaul später, dass dieser ihm mitgeteilt habe, dass nur die Güter in Österreich, nicht aber jene in der Steiermark auf die Witwe übergehen sollten. ${ }^{167}$ Apollonia vermachte das Gut nun eben diesem Bruder, davon sollte er ihren Schwestern und ihrem Neffen Anton Puchheim je 300 Gulden ausbezahlen, sofern diese ihn im Falle einer gerichtlichen oder anderen Infragestellung der Besitzrechte unterstützen würden. ${ }^{168}$ Einerseits lehrt das Beispiel, dass Verfügungen auch noch am Totenbett getroffen werden konnten, deren Durchsetzung aber nicht unproblematisch war. Andererseits fühlte sich Apollonia offensichtlich in den Besitzrechten noch nicht endgültig bestätigt, schloss zukünftige Konflikte nicht aus. Schließlich ist der aus dem Prozess stammende Hinweis auf die Aufbewahrung des Siegels durch die Ehefrau nicht uninteressant. Als Abschluss sei auf eine Verfügung von Margaretha Prockh verwiesen, die festlegte, dass im Fall von noch offenen bzw. unvollzogenen Verordnungen des Testaments ihres Mannes diese vollstreckt werden sollten. ${ }^{169}$

Doch mit dem Beispiel der Anweisungen und breit gefächerten Aufgaben der Exekutoren sowie allgemein der möglichen Schwierigkeiten der Durchsetzung von Verfügungen ist bereits eine Ebene erreicht, die nur durch weiterreichende Forschungen zur tatsächlichen Umsetzung, also ob und wann es Streitigkeiten gab,

167 Perner, $4^{\mathrm{r}}-6^{\mathrm{r}}$. POSCH, Hartberg 14.

168 Perner, $4^{\mathrm{v}}-5^{\mathrm{v}}$.

169 Prockh, $3^{\text {r }}$ diskutiert werden könnte und in dieser Arbeit eben bis auf wenige Schlaglichter ausbleiben muss.

Gehen wir einen Schritt zurück zur Ebene der Eröffnung und damit Einbringung des letzten Willens: Betrachtet man zunächst die Vorgaben der bereits genannten Landtafel, so legte diese fest, dass ein Testament nicht vom Erben selbst, sondern bei Mitgliedern des Herren- und Ritterstandes im Beisein der Verwandten innerhalb Monatsfrist verkündet werden sollte. Wurden darin Verfügungen zum Begräbnis getroffen, sollte es eröffnet, aber allein diese Punkte durch die anwesenden Verwandten und Erben zu Rate gezogen werden. Erzielte man wegen der Eröffnung des letzten Willens keine Einigung oder waren Beteiligte weit entfernt sesshaft bzw. der Aufenthaltsort u.U. sogar unbekannt, dann konnte der instituierte Erbe den Landeshauptmann bitten, einen Termin festzulegen und die Betroffenen durch Befehlschreiben oder Patente zu informieren. ${ }^{170}$ Am besagten Tag wurde dann auch bei Nichterscheinen von Vorgeladenen das Testament verlesen. In jedem Fall wurde der letzte Wille bei einem verschlossenen Dokument erst durch den Akt des Öffnens und Verlesens rechtswirksam. ${ }^{171}$ Auch wenn der Erbe, Exekutor oder allgemein der Verwahrer des Testaments dieses zu lang zurückhielt, konnten Verwandte und Begünstigte die Landesobrigkeit um Hilfe bei Festsetzung eines Termins bitten. Eine Einbeziehung der Landesinstanzen erfolgte bei den vorliegenden Testamenten etwa im Fall der Anna Kannibal (s. unten). Auch nichtverschlossene Testamente sollten im Beisein der

\footnotetext{
${ }^{170}$ Diese Möglichkeit eines Eingriffs der Landesobrigkeit wurde insofern erweitert, da auf Bitte von Verwandten, Gläubigern oder anderen Betroffenen der Landeshauptmann eine Deklarierung des Haupterben zum Antritt in einer Zeitspanne von mindestens einem halben Jahr einfordern konnte (STRÄTZ, Landtafel Titel 21, § 3, 354).

${ }^{171}$ Vgl. zum Erbschaftsantritt allg. WESENER, Erbrecht 23-27.
} 
Verwandten innerhalb der gesetzten Frist verlesen werden, auch wenn dieses bei Nichtausführung dann kraftlos wurde. Allgemein galt, dass wenn der vorgeschriebene Rahmen zur Bekanntgabe nicht eingehalten werden konnte, das Testament nicht ungültig wurde. Zusätzlich waren bei der Eröffnung auch die Zeugen, wie dann im Fall der Kannibal, einzuladen, damit diese ihr Siegel und ihre Unterfertigung bestätigen konnten. Dabei musste lediglich ein Großteil dieser anwesend sein, ein Fernbleiben aus gutem Grund oder der Tod eines der Zeugen machten das Testament nicht ungültig. ${ }^{172}$ Auch hier kann auf den Fall der Anna Kannibal verwiesen werden.

Betrachtet man die vorliegenden Fallbeispiele, so verlangte Margaretha Prockh explizit, dass das Testament beim Landmarschall eingebracht werden sollte, zumal dieser die zuständige $\mathrm{Ob}$ rigkeit darstelle, da sie keine bürgerlichen Güter in Wien besitze. Diese klare Formulierung war wohl deshalb notwendig, da ihr Mann, Hans Prockh, auch Wiener Bürger war. ${ }^{173}$ Besonders aufschlussreich sind für die Bekanntgabe der letzten Willen die bei einigen Testamenten vorhandenen Beilagen. Sehr gut lässt sich dieser Prozess anhand der dem Testament der Anna Kannibal beigelegten Schriftstücke nachvollziehen: Am 25. August 1567 ersuchte Cristoph Kannibal, der Sohn der Verstorbenen, in einem Schreiben an den Landmarschall um eine baldige Öffnung des Testaments, da seine Mutter bereits seit einem Jahr verstorben sei, viele Gläubiger Ansprüche angemeldet hätten und die Lehenschaft innerhalb der Jahresfrist heimfallen werde. Dieser benannte in der Folge drei weitere Personen, darunter einen Anwalt zu Kirchberg, die das Testament öffnen und vor dem Witwer und den Kindern vortragen sollten. Darauf verfasste der Landmarschall Hans Wil-

172 STRÄTZ, Landtafel Titel 20, 352f.

173 Prockh, 9v. Zu Wien als Gerichtsstand vgl. kompakt HOCHEDLINGER, Archivarischer Vandalismus 314f. helm von Roggendorf 174 ein Schreiben an die Genannten, dem er die Supplik beilegte und in dem er die Bitte zur Bildung der Kommission billigte. Diese sollte einen Tag zur Verkündung des letzten Willens bestimmen und dies im Beisein der Parteien ausführen sowie dann in einem Schreiben an den Landmarschall den Vorgang darstellen. Tatsächlich geschah das in einem Schriftstück vom 22. September 1567 aus Gmünd. Das Schriftstück wurde von zwei der durch Cristoph Kannibal genannten Personen unterzeichnet. Darin betonten sie die rechtzeitige Ausschreibung der Testamentseröffnung, das Erscheinen der Zeugen, wobei mittlerweile einer verstorben war, und das des Cristoph Kannibal, der für sich selbst sowie seine drei Schwestern auftrat. Deren Vater Anton Kannibal blieb jedoch aus. Das Testament wurde im Beisein der Erschienenen eröffnet, Cristoph Kannibal bat um Abschriften, da das Original dem Schreiben für den Landmarschall beigelegt wurde. ${ }^{175}$ Ein Erledigungsvermerk vom 4. Dezember 1567 unter der auf der Rückseite des Schreibens angebrachten Außenadresse verwies ebenfalls auf das Original des Testaments, das bei Gericht aufbewahrt werden sollte. In jedem Fall belegt dieses Beispiel, dass das Verlesen des letzten Willens für den Erwerb der Erbschaft notwendig war.

In den anderen Fällen sind es kurze Hinweise auf die Öffnung des Testamentes, die aber auch nur selten vorhanden sind: So wurde das Testament von Margaretha Prockh am 15. April 1567 im Haus das Landuntermarschalls geöffnet, also nur sechs Tage nach Beglaubigung des Testaments, was angesichts der bereits erwähn-

\footnotetext{
${ }^{174} \mathrm{Vgl}$. zu diesem und seiner Rolle bei der Reformation REINGRABNER, Adel 32-34.

${ }^{175}$ Die Zustellung von Abschriften an die Parteien wird auch in einem Vermerk unter dem Rubrum auf der Außenseite des Testaments des Josua Hollgruber befohlen (17. 10. 1541, HHStA, NÖLMA 13, Nr. 100).
} 
ten Leibsschwachheit nicht verwunderlich ist. ${ }^{176}$ Der letzte Wille der Elisabeth Nagl wurde erst 1585 verkündet; nur noch der Sohn und keiner der Zeugen war bei diesem Vorgang anwesend. Eine Beilage außerhalb des Jahrzehnts dieser Arbeit, aber für diese Fragestellung wichtig, befand sich bei dem Testament des Josua Hollgruber. In diesem Schreiben an den Landmarschall teilte einer der Zeugen mit, dass er das Testament des bereits verstorbenen Erblassers aufbewahrt habe und auf dessen Wunsch dieses nun der Landkanzlei übersende. ${ }^{177}$

Ein letzter Vorgang kann anhand dreier Beispiele von Testamenten für die Zeit um die Mitte des 16. Jahrhunderts belegt werden, nämlich das Eintragen in ein Testamentsbuch. So verlangte Barbara Pösing von ihren Exekutoren, dass ihr letzter Wille „zu ewiger gedechtnus in daz landt prothocoll oder testament buch ordentlich eingeschriben werde".178 Ein Vermerk auf der Rückseite unterhalb des Rubrums eines älteren Testaments von 1540 erwähnte die vorzunehmende Eintragung des Dokumentes in ein Landestestamentsgedenkbuch. Der Testator selbst bat einen Sekretär der Landschaft in seinem letzten Willen auch darum, wobei er es als Landbuch bezeichnete ${ }^{179}$. Am Ende des Testaments des Georg von Königsberg (5. November 1548) ist folgendes zu lesen: „Collationirt durch mich Johan Ferber, verwalter dess lanndtschreiberambts und dem originall wie daselb in das landgedenckbuech eingeschriben von wort zu wort also gleich lauttenndt befunden." 180 Dies belegt wiederum die Existenz eines Testamentsbuches, v.a. aber dessen hohen rechtlichen Wert, da dieses auch zur Herstellung von Abschriften

${ }^{176}$ Der Vermerk befindet sich unter dem auf der Rückseite der letzten Seite der Ausfertigung angebrachten Rubrum.

177 HHStA, NÖLMA 13, Nr. 100 (Hollgruber).

178 Pösing, 12v .

${ }^{179}$ HHStA, NÖLMA 22, Nr. 40 (Wolfgang

Mospeck), $1^{\mathrm{v}}$.

${ }^{180}$ HHStA, NÖLMA 17, Nr. 31 (Georg Königsberg), 4 r . herangezogen wurde, dabei sogar als Original galt. Dieses dürfte auch zur Festhaltung durch Zeugen vorgebrachter mündlicher Testamente verwendet worden sein, wobei man auch die rechtliche Qualität von davon vorgenommenen Abschriften betonte. ${ }^{181}$

Zwar sind es nur Schlaglichter, die uns Informationen zur Testamentsvollstreckung geben, auch werden die einzelnen Vorgänge nicht immer gleich verlaufen sein, immerhin erhält man in Zusammenschau mit den rechtlichen, etwas jüngeren Vorgaben aber einen Eindruck von den vorhandenen Möglichkeiten.

\section{Zusammenfassung}

Ziel dieser Arbeit war es, einen Einblick in die adlige Testamentspraxis der frühen zweiten Hälfte des 16. Jahrhunderts zu geben. Dabei sollte der Bogen von den äußeren Testamentsformen über deren inhaltliche Merkmale zu dem Umgang mit den Stücken in der Praxis gespannt werden. Trotz der unterschiedlichen Testatoren sind in dem Aufbau der Stücke und der Verwendung der Formeln durchaus Parallelen zu erkennen. Eine allgemeine Struktur konnte erschlossen werden. Zudem wurde anhand weniger Hinweise der Vorgang der Entstehung der Dokumente bis $\mathrm{zu}$ deren Umsetzung verfolgt, was die Bedeutung der Quelle auch abseits von Fragen der Realienkunde und Mentalitätsgeschichte belegt. Nicht uninteressant ist die Tatsache, dass die Praxis durchaus in einigen Punkten Gemeinsamkeiten mit der Jahrzehnte später verfassten Landtafel Österreichs ob der Enns erkennen lässt. Besonders bemerkenswert scheint der anhand der Dokumente erfolgte Nachweis des ehemaligen Vorhandenseins eines Testamentsbuches bei der Landschaft.

Themen wie die Form und Art der Legate (Realien), die Aufteilung des Erbes, Fragen nach

${ }^{181}$ Zitat nach Püdler in WESENER, Erbrecht 140. 
Hinweisen auf Konfession in diesem bedeutsamen Jahrzehnt, persönliche Elemente oder auch Zusammenhänge der Testamente selbst konnten in diesem vorgegebenen Rahmen nicht erörtert werden, doch wird diesen Fragen an anderer Stelle nachgegangen werden.

\section{Literatur:}

Beatrix BASTL, Tugend, Liebe, Ehre. Die adelige Frau in der Frühen Neuzeit (Wien-Köln-Weimar 2000).

Evelyn BENESCH (Bearb.), Die Kunstdenkmäler Österreichs. Niederösterreich nördlich der Donau (DEHIO-Handbuch, Wien 1990).

Rudolf BÜTTNER, Burgen und Schlösser an der Donau (Wien 1964).

Peter CsENDES, Die Rechtsquellen der Stadt Wien (= Fontes Rerum Austriacarum, 3. Abt.: Fontes Iuris, Bd. 9, Wien-Köln-Graz 1986).

Anton EgGENDORFER, Das Viertel ober dem Manhartsberg im Spiegel des Bereitungsbuches von 1590/91, Bd. 1 (phil. Diss., Univ. Wien 1974).

Adalbert ERLER, Vormundschaft, in: HRG ${ }^{1}$, Bd. 5 (Berlin 1998) 1050-1055.

Mattias Gerhard FISCHER, Zeugen, in: $\mathrm{HRG}^{1}$, Bd. 5 (Berlin 1998) 1684-1693.

Karl Friedrich von FRANK, Standeserhebungen und Gnadenakte für das Deutsche Reich und die Österreichischen Erblande bis 1806 sowie kaiserlich österreichische bis 1823, 5 Bde. (Senftenegg 1967-1974).

Jacob und Wilhelm GRIMM, Deutsches Wörterbuch, 33 Bde. (München 1854-1971, Nachdruck 1991).

Hans-Rudolf HAGEMANN, Erbrecht, in: $\mathrm{HRG}^{2}$, Bd. 1 (Berlin 2008) 1370-1384.

Herbert HAUSMANINGER, Walter SELB, Römisches Privatrecht (Wien-Köln-Weimar ${ }^{92001) .}$

Michael HocHEDLINGER, Archivarischer Vandalismus? Zur Überlieferungsgeschichte frühneuzeitlicher Testamente und Verlassenschaftsabhandlungen in Österreich, in: Archivalische Zeitschrift 84 (2001) 289-364.

Michael HocHEDLINGER, Aktenkunde. Urkunden- und Aktenlehre der Neuzeit (Wien-München 2009).

Hans KIEFNER, Rezeption (privatrechtlich), in: HRG ${ }^{1}$, Bd. 3 (Berlin 1984) 970-984.

Johann Evang(elist) KIRNBAUER VON ERZSTÄTT (Bearb.), Siebmachers Wappenbuch, Bd. IV, Abt. 4: Der Niederöstereichische Adel, 1. Teil (Nürnberg 1909 = Bd. 26 des ND Neustadt an der Aisch 1983).
Karl LeCHNER (Hg.), Donauländer und Burgenland. Handbuch der historischen Stätten Österreich, Bd. 1 (Stuttgart 1970).

Martin LIPP, Erbfolgeordnung, in: $\mathrm{HRG}^{2}$, Bd. 1 (Berlin 2008) 1361-1365.

Werner OGRIS, Geschäftsfähigkeit, in: $\mathrm{HRG}^{1}, \mathrm{Bd} .1$ (Berlin 1971) 1594-1596.

Werner OGRIS, Nachlass, in: HRG ${ }^{1}$, Bd. 3 (Berlin 1984) $820-823$.

Werner OGRIS, Noterben, in: HRG ${ }^{1}$, Bd. 3 (Berlin 1984) 1056-1059.

Werner OGRIS, Testament, in: HRG ${ }^{1}$, Bd. 5 (Berlin 1998) 152-165.

Richard Perger, Die Aufnahme von Wiener Bürgern in den Ritterstand Österreichs unter der Enns im 16. und frühen 17. Jahrhundert, in: Jahrbuch für Landeskunde von Niederösterreich NF 63/64 (1998) 365-376.

Walter PONGRATZ, Gerhard SEEBACH, Burgen und Schlösser in Niederösterreich, Bd. III/2. YsperPöggstall-Weiten (Wien 1972).

Fritz PosCH, Geschichte des Verwaltungsbezirkes Hartberg, Bd. 2: Historisch-Topographischer Teil (Graz 1990).

Gustav REINGRABNER, Adel und Reformation (=Forschungen zur Landeskunde von Niederösterreich 21, Wien 1976).

Helene SAlzMANN, Maximilian I. und die Herren von Polheim (phil. Diss., Univ. Wien 1967).

Ruth SCHMIDT-WIEGAND, Vormund, in: HRG ${ }^{1}$, Bd. 5 (Berlin 1998) 1049-1050.

Silvia SCHODL, Die Zusammensetzung des niederösterreichischen Ritterstandes in der Zeit von 1568 bis 1620 (phil. Diss., Univ. Wien 1983).

Lutz SEDATIS, Universalsukzession, in: $\mathrm{HRG}^{1}, \mathrm{Bd} .5$ (Berlin 1998) 490-492.

Hans-Wolfgang STRÄTZ (Bearb.), Landtafel Österreich ob der Enns, Bd. 1: Verfaßte Landtafel von 1616 und corrigierte Landtafel von 1629 (= Forschungen zur Geschichte Oberösterreichs 17, Linz 1990).

Max VANCSA, Topographie von Niederösterreich, Bd. 6: Alphabetische Reihenfolge und Schilderung der Ortschaften in Niederösterreich (Wien 1909).

Alois WeISS vON STARKENFELS, Johann Evang(elist) KIRNBAUER VON ERZSTÄTT (Bearb.), Siebmachers Wappenbuch. Bd. IV, Abt. 5: Der Oberösterreichische Adel (Nürnberg 1904 = Bd. 27 des ND Neustadt an der Aisch 1984).

Moriz Wertner, Die Grafen von St. Georgen und Bösing, in: Jahrbuch „Adler“ NF 1 (1891) 171-235. 
Gunter WESENER, Geschichte des Erbrechtes in Österreich seit der Rezeption (= Forschungen zur Neueren Privatrechtsgeschichte 4, Graz-Köln 1957).

Theodor WiEDEMANN, Geschichte der Reformation und Gegenreformation im Lande unter der Enns, Bd. 2 (Prag 1880).

Johann Baptist WiTTING (Bearb.), Siebmachers Wappenbuch, Bd. IV, Abt. 4: Der Niederöstereichische Adel, 2. Teil (Nürnberg $1918=$ Bd. 26 des ND Neustadt an der Aisch 1983).

Andreas ZAJIC, "Zu ewiger gedächtnis aufgericht". Grabdenkmäler als Quelle für Memoria und Repräsentation von Adel und Bürgertum im Spätmittelalter und der Frühen Neuzeit. Das Beispiel Niederösterreichs (= MIÖG Erg.Bd. 45, WienMünchen 2004).
Andreas ZAJIC, Die Inschriften des politischen Bezirks Krems (=Die deutschen Inschriften 72, Wien 2008).

Alois ZAUNER, Vöcklabruck und der Attergau. Stadt und Grundherrschaft in Oberösterreich bis 1620 (Linz 1971).

\section{Abkürzungen:}

NÖLMA Niederösterreichisches Landmarschallamt

Siehe auch das allgemeine Abkürzungsverzeichnis: http://www.rechtsgeschichte.at/beitraege/abk.pdf 


\begin{tabular}{|c|c|c|c|c|c|c|c|c|c|c|c|c|c|c|}
\hline$\sum_{j}^{\S}$ & 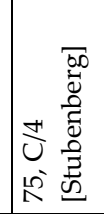 & 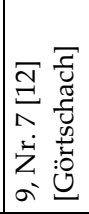 & 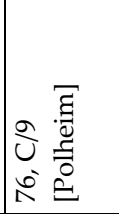 & 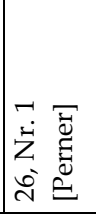 & 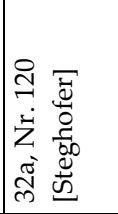 & 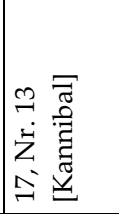 & 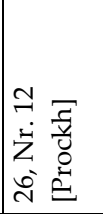 & 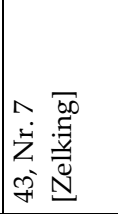 & 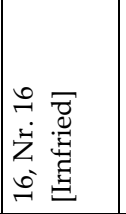 & 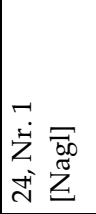 & 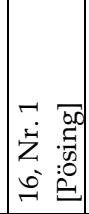 & 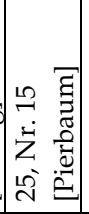 & 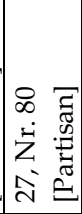 & 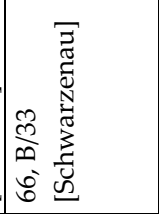 \\
\hline 壳 & 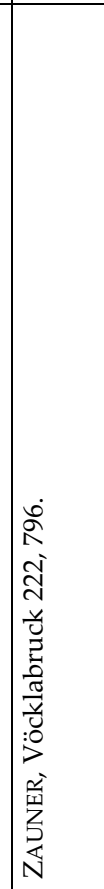 & 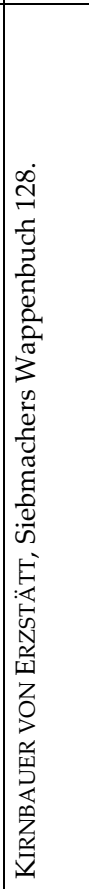 & 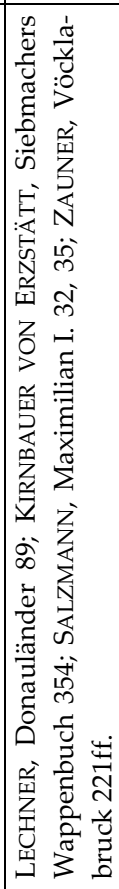 & 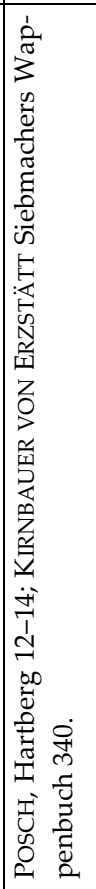 & 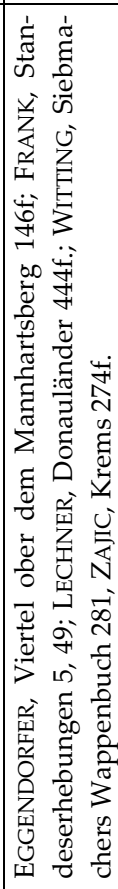 & 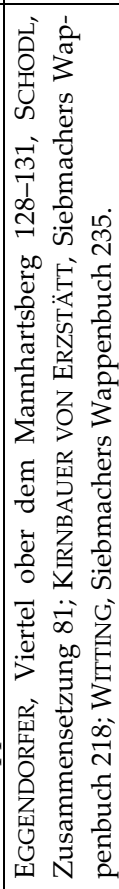 & 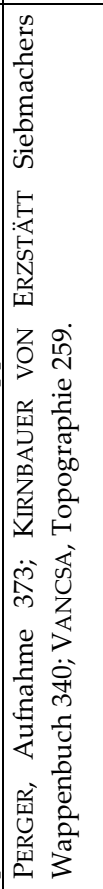 & 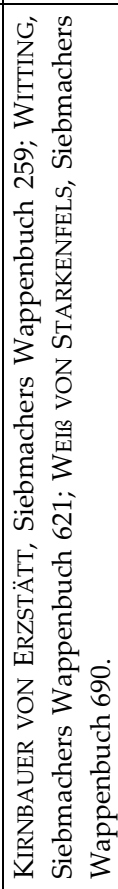 & 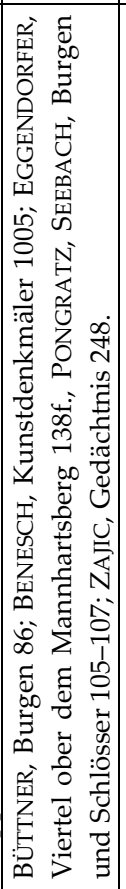 & 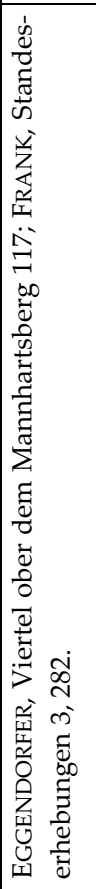 & 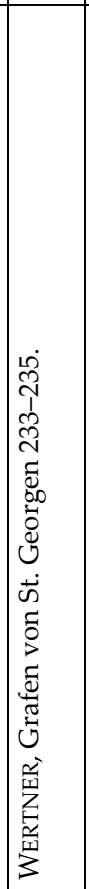 & 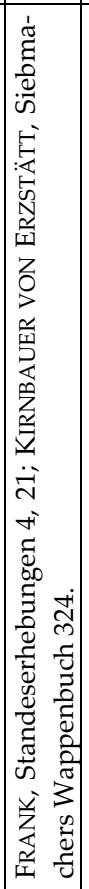 & & 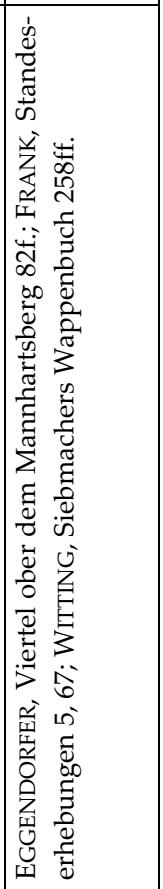 \\
\hline 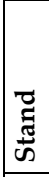 & 离 & 竧 & 烒 & 烒 & 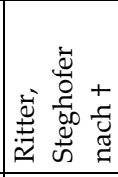 & 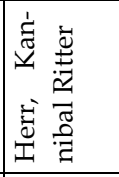 & \begin{tabular}{|l} 
离 \\
壳
\end{tabular} & 莣 & \begin{tabular}{|l} 
离 \\
壳
\end{tabular} & 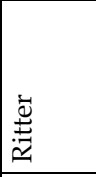 & 烒 & 竧 & r. & 竞 \\
\hline 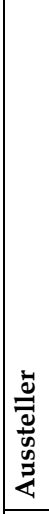 & 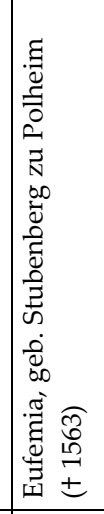 & 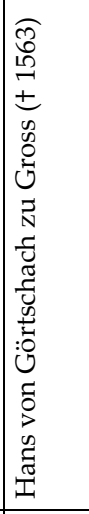 & 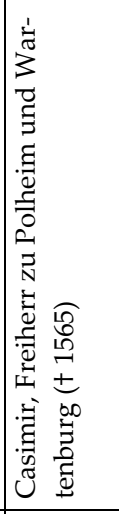 & 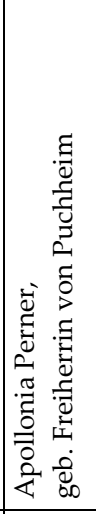 & 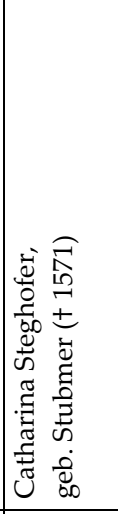 & 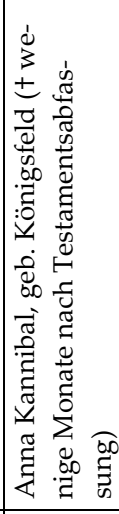 & 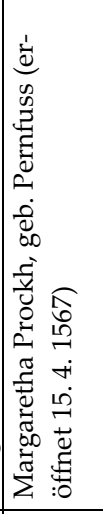 & 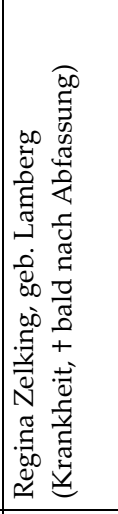 & 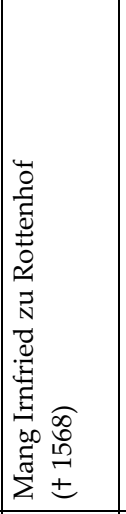 & 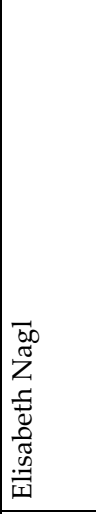 & 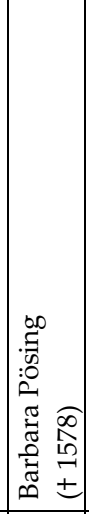 & 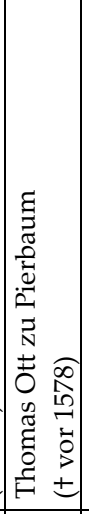 & 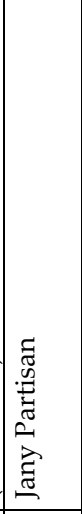 & 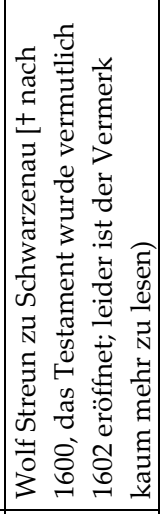 \\
\hline 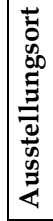 & 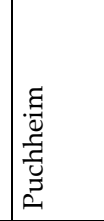 & $\begin{array}{l}0 \\
0 \\
0 \\
0 \\
0\end{array}$ & 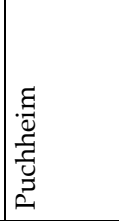 & 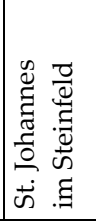 & 岳 & 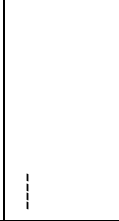 & $\sum_{3}^{\tilde{D}}$ & ${ }_{3}^{\frac{5}{3}}$ & 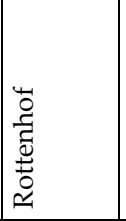 & 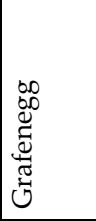 & $\overbrace{3}^{\tilde{0}}$ & 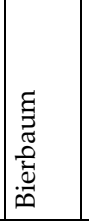 & 离 & 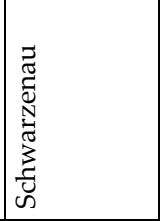 \\
\hline ص్ & 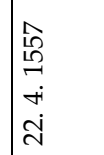 & 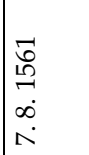 & 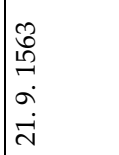 & 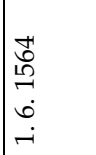 & 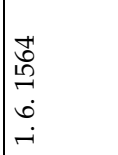 & 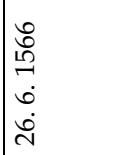 & 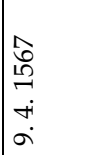 & 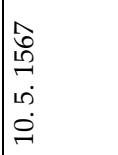 & 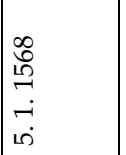 & 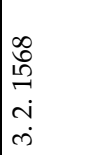 & $\begin{array}{l}0 \\
0 \\
0 \\
0 \\
\infty \\
0 \\
0 \\
0\end{array}$ & 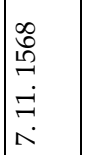 & 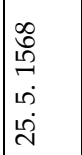 & 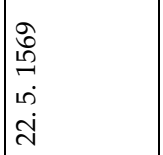 \\
\hline
\end{tabular}

